Article

\title{
Two-Way Multi-Antenna Relaying with Simultaneous Wireless Information and Power Transfer
}

\author{
Thanaphat Srivantana and Kiattisak Maichalernnukul * \\ College of Information and Communication Technology, Rangsit University, Pathumthani 12000, Thailand; \\ aof-rangsit@hotmail.com \\ * Correspondence: kiattisak.m@rsu.ac.th \\ Academic Editor: Angel Garrido \\ Received: 24 December 2016; Accepted: 14 March 2017; Published: 16 March 2017
}

\begin{abstract}
In this paper, we propose various kinds of two-way multi-antenna relaying with simultaneous wireless information and power transfer (SWIPT) and investigate their performance. Specifically, we first consider a two-way relay network where two single-antenna end nodes communicate with each other through a multi-antenna relay node that is energy constrained. This relay node harvests energy from the two end nodes and use the harvested energy for forwarding their information. Six relaying schemes that support the considered network then build on the power splitting-based relaying and time switching-based relaying protocols. The average bit error rates of these schemes are evaluated and compared by computer simulations considering several network parameters, including the number of relay antennas, power splitting ratio, and energy harvesting time. Such evaluation and comparison provide useful insights into the performance of SWIPT-based two-way multi-antenna relaying.
\end{abstract}

Keywords: amplify-and-forward; bit error rate; decode-and-forward; network coding; simultaneous wireless information and power transfer (SWIPT); space-time coding; two-way multi-antenna relaying

\section{Introduction}

Recently, simultaneous wireless information and power transfer (SWIPT) has gained great interest due to its capability to deal with the energy scarcity in energy-constrained wireless networks [1-10]. In the seminal works [1,2], the fundamental trade-off between information and power transfer in different point-to-point wireless channels was studied. On the other hand, two practical receiver designs for SWIPT, namely power splitting (PS) and time switching (TS), were firstly presented in [3,4]. Specifically, the PS-based receiver spits the received radio-frequency signal into two streams of different power for harvesting energy and decoding information, whereas the TS-based receiver switches over time between those two operations. The SWIPT has been adopted later in more complicated communication scenarios, including the broadband wireless system [5], the cellular network [6], the interference channel $[7,8]$, and the relay channel $[9,10]$. This paper focuses on the last scenario.

Many works in the literature (e.g., [11-15]) have been devoted to two-way multi-antenna relaying (without SWIPT) as this approach can not only extend communication range but also improve spectral efficiency. In a basic two-way multi-antenna relay network (see Figure 1), an intermediate relay node equipped with multiple antennas is used to assist two end nodes in exchanging their information. Nevertheless, application of SWIPT to this kind of network is still in its infancy [16-18]. In [16], the SWIPT-based beamforming design for a multi-antenna relay was considered to maximize the sum rate of its two-way relay network. In [17], the authors presented a three-phase two-way relay network where an energy-constrained multi-antenna relay node harvests energy from a pair of single-antenna source nodes, and presented an optimal power allocation solution. In [18], an optimal joint source 
and relay beamforming scheme for two-way multi-antenna relay networks with SWIPT was proposed based on the principle of singular value decomposition.

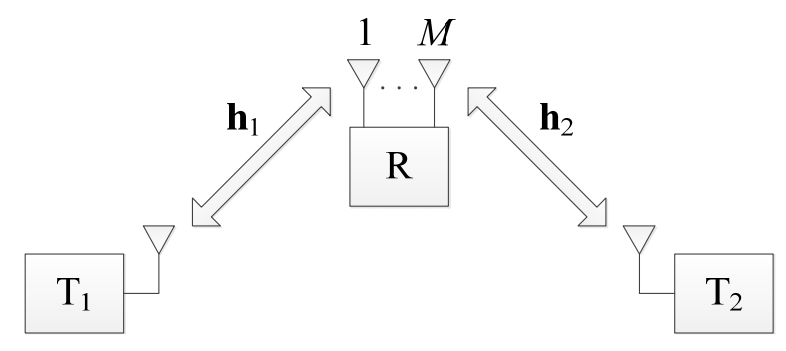

Figure 1. System model.

Depending on the nature and complexity of relays, relaying schemes can be classified into two main categories: non-regenerative relaying and regenerative relaying [19]. Non-regenerative relaying generally implies that the relays only amplify their received signals before retransmitting them. Then, it is often referred to as amplify-and-forward (AF) relaying in the literature. Note that all existing works on two-way multi-antenna relaying with SWIPT (i.e., [16-18]) are non-regenerative. On the other hand, regenerative relaying requires the relays to change the waveforms and/or the data contents by performing some processing in the digital domain. An example is the decode-and-forward (DF) relay, which receives the data from its immediate predecessor, decodes, re-encodes, and finally retransmits it. To the best of our knowledge, regenerative relaying has not yet been considered in the SWIPT-based two-way multi-antenna relay networks.

In this paper, we consider a two-way relay network in which two single-antenna end nodes communicate with each other through a multi-antenna relay node that is energy constrained. This relay node harvests energy from the two end nodes and use the harvested energy for forwarding their information. Based on two half-duplex relaying protocols, called power splitting-based relaying (PSR) and time switching-based relaying (TSR) [9], for separate energy harvesting and information processing at the relay node, six multiple-antenna relaying schemes, namely PS-AF, PS-DF, PS-DF with space-time coding (PS-DF-STC), TS-AF, TS-DF, and TS-DF-STC, are designed for the considered network. In the DF-oriented design, network coding (NC) [20] is applied to the end nodes' information that is decoded at the relay node. Moreover, by having multiple antennas at the relay node, STC [21] is used in the PS-DF-STC and TS-DF-STC schemes with the aim of achieving a better end-to-end decoding performance. Unlike the aforementioned works [16-18], which are devoted to analyzing the relevant sum-rate performance, this paper will investigate the average bit error rates (BERs) of the proposed relaying schemes as a function of the number of relay antennas, power splitting ratio, and energy harvesting time.

The remainder of this paper is organized as follows. Section 2 introduces the system model. Sections 3 and 4 present the PS-based and TS-based multiple-antenna relaying schemes, respectively. Section 5 compares the BER performance of these relaying schemes by simulations. Finally, Section 6 concludes the paper.

Notation: Bold upper-case letters denote matrices and bold lower-case letters denote column vectors. $[\cdot]_{i j},(\cdot)^{\mathrm{T}},(\cdot)^{\mathrm{H}}$, and $(\cdot)^{-1}$ denote the $(i, j)$-th element, transpose, conjugate transpose, and inverse of a matrix, respectively. $\bmod (\cdot)$ and $\operatorname{demod}(\cdot)$ denote the modulation and demodulation functions, respectively. $|\cdot|, \mathrm{E}[\cdot]$, and $\oplus$ denote the absolute value, the expectation value, and the bit-wise exclusive OR operator, respectively. $Q(\cdot), \operatorname{erfc}(\cdot), \Gamma(\cdot)$, and ${ }_{2} \mathrm{~F}_{1}(\cdot, \cdot ; \cdot ; \cdot)$ denote the Gaussian Q-function, complementary error function, ordinary Gamma function, Gauss hypergeometric function defined in (Equation (4.1), [22]), (p. xxxvi, [23]), (Equation (8.310.1), [23]), and (Equation (9.100), [23]), respectively. A circularly symmetric complex Gaussian random variable $z$ with mean $\mu$ and variance $\sigma^{2}$ is denoted as $z \sim C N\left(\mu, \sigma^{2}\right)$. In the case of communication over a slow-fading channel, the average BER for binary phase shift keying (BPSK) modulation is defined by (Equation (5.1), [22]) 


$$
\bar{P}_{\mathrm{e}}=\int_{0}^{\infty} Q(\sqrt{2 x}) f \gamma(x) d x
$$

where $f \gamma(x)$ is the probability density function (PDF) of the instantaneous signal-to-noise ratio (SNR) of the received signal, $\gamma$.

\section{System Model}

Consider a two-way relay network as shown in Figure 1, where end nodes $T_{1}$ and $T_{2}$, each of which is equipped with one antenna, exchange information through an energy-constrained intermediate relay node, $\mathrm{R}$, possessing $M$ antennas. This relay node will harvest energy from the two end nodes and use the harvested energy for forwarding their information. The relay node's antennas are spatially spaced in such a way that the received/transmitted signals undergo statistically independent fading. Throughout this paper, perfect timing and synchronization among $\mathrm{T}_{1}, \mathrm{~T}_{2}$, and $\mathrm{R}$ are assumed, and BPSK modulation is used at $\mathrm{T}_{1}$ and $\mathrm{T}_{2}$. Let $h_{1, m} \sim C N\left(0, \sigma_{1}^{2}\right)$ (or $h_{2, m} \sim C N\left(0, \sigma_{2}^{2}\right)$ ) denote the channel gain between the antenna of $\mathrm{T}_{1}$ (or $\mathrm{T}_{2}$ ) and the $m$-th antenna of $\mathrm{R}$, where $\sigma_{1}^{2}=d_{1}^{-v}$ (or $\sigma_{2}^{2}=d_{2}^{-v}$ ) is due to the path loss with power path loss exponent $v$ and distance $d_{1}\left(\right.$ or $d_{2}$ ) of the $\mathrm{T}_{1}-\mathrm{R}$ link (or the $\mathrm{T}_{2}-\mathrm{R}$ link), and $m=1,2, \ldots, M$. We presume that all the channels are static over an interval of $2 N$, which denotes the total block time in which a certain block of information is exchanged between $T_{1}$ and $T_{2}$ (see Figures 2a and 3a), and ignore the direct link between the end nodes owing to the larger distance compared with the $\mathrm{T}_{1}-\mathrm{R}$ and $\mathrm{T}_{2}-\mathrm{R}$ links [16]. For analytical simplicity, we assume that the relay node is located halfway between the end nodes, and thus $\sigma_{1}^{2}=\sigma_{2}^{2}=\sigma^{2}$. As mentioned in Section 1, we consider two half-duplex relaying protocols for separate energy harvesting and information processing at the relay node, i.e., PSR and TSR.

\section{PSR Protocol}

Figure 2 illustrates the key parameters in the PSR protocol for energy harvesting and information processing at the relay node $\mathrm{R}$ and the block diagram of the corresponding receiver. In Figure $2 \mathrm{a}$, the first block time $N$ is used for multiple access (MA) where the end nodes $\mathrm{T}_{1}$ and $\mathrm{T}_{2}$ transmit their signals simultaneously, and $P$ is the total signal power. In the second block time $N$, the relay node processes this signal (according to the schemes that will be presented below) and broadcasts it. During the MA phase, the fraction of the received signal power $\rho P$ is used for energy harvesting, and the remaining received power $(1-\rho) P$ is used for information transmission, where $0<\rho<1$ is the power splitting ratio. In other words, as shown in Figure $2 b$, the portion of the received radio-frequency (RF) signal, denoted by $\sqrt{\rho} y_{\mathrm{r}, m}$, is sent to the energy harvesting receiver and the remaining signal strength, denoted by $\sqrt{1-\rho} y_{\mathrm{r}, m}$, drives the information receiver. In the following, we describe three multiple-antenna relaying schemes, which correspond to the PSR protocol.

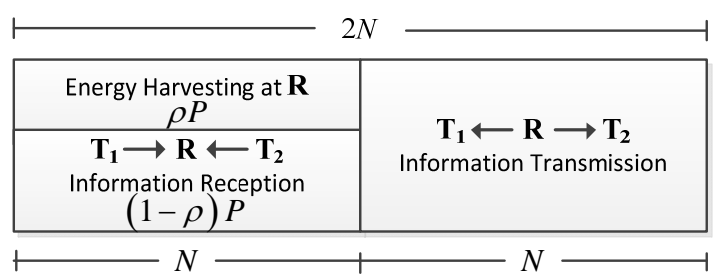

(a)

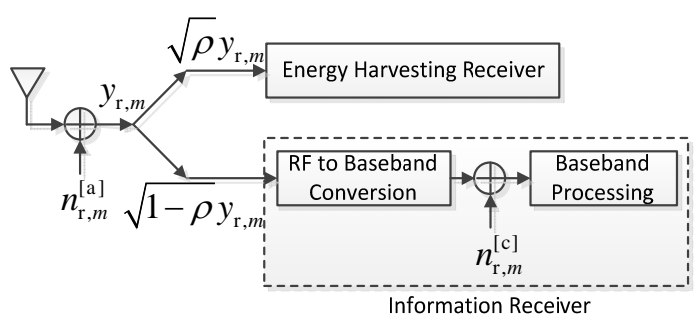

(b)

Figure 2. (a) Key parameter in the PSR protocol for energy harvesting and information processing at the relay node; and (b) Block diagram of the relay receiver (with a focus on its $m$-th antenna) in the PSR protocol. 


\subsection{PS-DF Scheme}

In the MA phase, the received RF signal at the relay node can be modeled as:

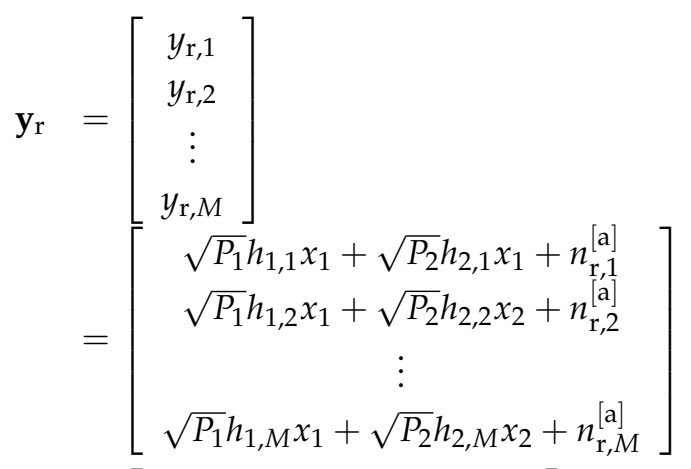

$$
\begin{aligned}
& =\left[\begin{array}{cc}
\sqrt{P_{1}} h_{1,1} & \sqrt{P_{2}} h_{2,1} \\
\sqrt{P_{1}} h_{1,2} & \sqrt{P_{2}} h_{2,2} \\
\vdots & \vdots \\
\sqrt{P_{1}} h_{1, M} & \sqrt{P_{2}} h_{2, M}
\end{array}\right]\left[\begin{array}{l}
x_{1} \\
x_{2}
\end{array}\right]+\left[\begin{array}{c}
n_{\mathrm{r}, 1}^{[\mathrm{a}]} \\
n_{\mathrm{r}, 2}^{[\mathrm{a}]} \\
\vdots \\
n_{\mathrm{r}, M}^{[\mathrm{a}]}
\end{array}\right] \\
& =\left[\begin{array}{ll}
\sqrt{P_{1}} \mathbf{h}_{1} & \sqrt{P_{2}} \mathbf{h}_{2}
\end{array}\right]\left[\begin{array}{l}
x_{1} \\
x_{2}
\end{array}\right]+\mathbf{n}_{\mathrm{r}}^{[\mathrm{a}]}
\end{aligned}
$$

where $P_{1}=\zeta_{1} P$ and $P_{2}=\zeta_{2} P$ are the transmitted power from $\mathrm{T}_{1}$ and $\mathrm{T}_{2}$, respectively; $0<\zeta_{1}, \zeta_{2}<1$ are the power ratios of $\mathrm{T}_{1}$ and $\mathrm{T}_{2}$, respectively (i.e., $\zeta_{1}+\zeta_{2}=1$ ); $x_{1}$ and $x_{2}$ are the normalized information signals from $\mathrm{T}_{1}$ and $\mathrm{T}_{2}$, respectively (i.e., $\mathrm{E}\left[\left|x_{1}\right|^{2}\right]=\mathrm{E}\left[\left|x_{2}\right|^{2}\right]=1$ ); and $n_{\mathrm{r}, m}^{[\mathrm{a}]} \sim \mathrm{CN}\left(0, \sigma_{\mathrm{a}}^{2}\right)$ is the additive white Gaussian noise (AWGN) at the $m$-th antenna of R. The energy harvesting receiver in Figure $2 \mathrm{~b}$ rectifies the RF signal $\sqrt{\rho} y_{\mathrm{r}, m}$ directly and gets the direct current to charge up the battery. Therefore, the harvested energy at the $m$-th antenna of the relay node during the MA phase is given by:

$$
E_{m}=\eta \rho N\left(P \sigma^{2}+\sigma_{\mathrm{a}}^{2}\right), m=1,2, \ldots, M
$$

where $0<\eta \leq 1$ is the energy conversion efficiency (which depends on the rectification process and the energy harvesting circuitry [9]). Meanwhile, the information receiver in Figure $2 b$ down-converts the RF signal $\sqrt{1-\rho} y_{\mathrm{r}, m}$ to baseband and processes the baseband signal, where $n_{\mathrm{r}, m}^{[\mathrm{c}]} \sim C N\left(0, \sigma_{\mathrm{c}}^{2}\right)$ is the AWGN due to RF-band-to-baseband signal conversion. After down conversion, the sampled baseband signal vector at the relay node is given by:

$$
\begin{aligned}
& \widetilde{\mathbf{y}}_{\mathrm{r}}=\sqrt{1-\rho} \mathbf{y}_{\mathrm{r}}+\mathbf{n}_{\mathrm{r}}^{[\mathrm{c}]} \\
& =\sqrt{1-\rho}\left(\left[\begin{array}{ll}
\sqrt{P_{1}} \mathbf{h}_{1} & \sqrt{P_{2}} \mathbf{h}_{2}
\end{array}\right]\left[\begin{array}{l}
x_{1} \\
x_{2}
\end{array}\right]+\mathbf{n}_{\mathrm{r}}^{[\mathrm{a}]}\right)+\mathbf{n}_{\mathrm{r}}^{[\mathrm{c}]} \\
& =\left[\begin{array}{ll}
\sqrt{(1-\rho) P_{1}} \mathbf{h}_{1} & \sqrt{(1-\rho) P_{2}} \mathbf{h}_{2}
\end{array}\right]\left[\begin{array}{l}
x_{1} \\
x_{2}
\end{array}\right]+\left(\sqrt{(1-\rho)} \mathbf{n}_{\mathrm{r}}^{[\mathrm{a}]}+\mathbf{n}_{\mathrm{r}}^{[\mathrm{c}]}\right) \\
& =\Psi\left[\begin{array}{l}
x_{1} \\
x_{2}
\end{array}\right]+\mathbf{n}_{r} .
\end{aligned}
$$

Assuming that $\rho, P_{1}, P_{2},\left\{h_{1, m}\right\}_{m=1}^{M}$, and $\left\{h_{2, m}\right\}_{m=1}^{M}$ are known at the relay node and applying zero-forcing (ZF) detection, estimates of $x_{1}$ and $x_{2}$, denoted by $\hat{x}_{1}$ and $\hat{x}_{2}$, respectively, are obtained as:

$$
\left[\begin{array}{c}
\hat{x}_{1} \\
\hat{x}_{2}
\end{array}\right]=\left(\Psi^{\mathrm{H}} \mathbf{\Psi}\right)^{-1} \boldsymbol{\Psi}_{\mathbf{y}}
$$


(For discussions of how the relay node can obtain $\left\{h_{1, m}\right\}_{m=1}^{M}$ and $\left\{h_{2, m}\right\}_{m=1}^{M}$, interested readers are referred to [24,25]).

Let $\gamma_{i r}$ denote the instantaneous SNR of $\hat{x}_{i}$, where $i=1,2$. It is straightforward to show that:

$$
\begin{aligned}
\gamma_{i \mathrm{r}} & =\frac{1}{\left((1-\rho) \sigma_{\mathrm{a}}^{2}+\sigma_{\mathrm{c}}^{2}\right)\left[\left(\mathbf{\Psi}^{\mathrm{H}} \mathbf{\Psi}\right)^{-1}\right]_{i i}} \\
& =\frac{(1-\rho) \zeta_{i} P \sum_{k=1}^{M-1} \sum_{l=k+1}^{M}\left|h_{1, k} h_{2, l}-h_{1, l} h_{2, k}\right|^{2}}{\left((1-\rho) \sigma_{\mathrm{a}}^{2}+\sigma_{\mathrm{c}}^{2}\right) \sum_{m=1}^{M}\left|h_{j, m}\right|^{2}} .
\end{aligned}
$$

expressed as:

Defining $\varphi:=\frac{\sum_{k=1}^{M-1} \sum_{l=k+1}^{M}\left|h_{1, k} h_{2, l}-h_{1, l} h_{2, k}\right|^{2}}{\sum_{m=1}^{M}\left|h_{j, m}\right|^{2}}$ and using (Equation (16), [26]), the PDF of $\varphi$ can be

$$
f_{\varphi}(x)=\frac{x^{M-2}}{\Gamma(M-1) \sigma^{2 M-2}} \exp \left(-\frac{x}{\sigma^{2}}\right) ; x \geq 0 .
$$

From Equations (6) and (7), we have:

$$
f_{\gamma_{\text {ir }}}(x)=\frac{\left((1-\rho) \sigma_{\mathrm{a}}^{2}+\sigma_{\mathrm{c}}^{2}\right)^{M-1}}{\Gamma(M-1) \sigma^{2 M-2}\left((1-\rho) \zeta_{i} P\right)^{M-1}} x^{M-2} \exp \left(-\frac{(1-\rho) \sigma_{\mathrm{a}}^{2}+\sigma_{\mathrm{c}}^{2}}{(1-\rho) \zeta_{i} P \sigma^{2}} x\right) ; x \geq 0 .
$$

Hence, the corresponding average BER can be derived as follows:

$$
\begin{aligned}
\bar{P}_{\mathrm{e}, i \mathrm{r}} & =\int_{0}^{\infty} Q(\sqrt{2 x}) f_{\gamma_{\mathrm{ir}}}(x) d x \\
& =\frac{1}{2 \sqrt{\pi}} \int_{0}^{\infty} \Gamma\left(\frac{1}{2}, x\right) f_{\gamma_{i \mathrm{r}}}(x) d x \\
& =\frac{\left((1-\rho) \sigma_{\mathrm{a}}^{2}+\sigma_{\mathrm{c}}^{2}\right)^{M-1}}{2 \sqrt{\pi} \Gamma(M-1) \sigma^{2 M-2}\left((1-\rho) \zeta_{i} P\right)^{M-1}} \\
& \times \int_{0}^{\infty} \Gamma\left(\frac{1}{2}, x\right) x^{M-2} \exp \left(-\frac{(1-\rho) \sigma_{\mathrm{a}}^{2}+\sigma_{c}^{2}}{(1-\rho) \zeta_{i} P \sigma^{2}} x\right) d x \\
& =\frac{\sigma \Gamma(M-1 / 2)\left((1-\rho) \zeta_{i} P\right)^{1 / 2}\left((1-\rho) \sigma_{\mathrm{a}}^{2}+\sigma_{\mathrm{c}}^{2}\right)^{M-1}}{2 \sqrt{\pi} \Gamma(M)\left((1-\rho)\left(\zeta_{i} P \sigma^{2}+\sigma_{\mathrm{a}}^{2}\right)+\sigma_{\mathrm{c}}^{2}\right)^{M-1 / 2}} \\
& \times{ }_{2} \mathrm{~F}_{1}\left(1, M-\frac{1}{2} ; M ; \frac{(1-\rho) \sigma_{a}^{2}+\sigma_{\mathrm{c}}^{2}}{(1-\rho)\left(\zeta_{i} P \sigma^{2}+\sigma_{\mathrm{a}}^{2}\right)+\sigma_{\mathrm{c}}^{2}}\right)
\end{aligned}
$$

where the second equality is obtained by using the fact that $Q(x)=\operatorname{erfc}(x / \sqrt{2}) / 2$ and (Equation (8.359.3), [23]), and the fourth equality is obtained by using (Equation (6.455.1), [23]).

The relay node then performs NC of $\hat{x}_{1}$ and $\hat{x}_{2}$ at bit level to obtain the composite signal. Specifically, let $\hat{b}_{i}=\operatorname{demod}\left(\hat{x}_{i}\right)$ be the estimated information bit sequence corresponding to $\hat{x}_{i}$, where $i=1,2$. The composite signal is given by $x_{\mathrm{r}}=\bmod \left(\hat{b}_{1} \oplus \hat{b}_{2}\right)$. As in [9], we assume that the processing power required by the transmit/receive circuitry at the relay node is negligible as compared to the power used for transmitting the composite signal in the broadcast (BC) phase. From Equation (3), the latter power is given by:

$$
\begin{aligned}
P_{\mathrm{r}} & =\frac{\sum_{m=1}^{M} E_{m}}{N} \\
& =\eta \rho M\left(P \sigma^{2}+\sigma_{\mathrm{a}}^{2}\right)
\end{aligned}
$$

and the sampled received (baseband) signal at the end node $\mathrm{T}_{i}(i=1,2)$ in the $\mathrm{BC}$ phase can be expressed as:

$$
y_{i}=\sqrt{P_{\mathrm{r}}} \sum_{m=1}^{M} h_{i, m} x_{\mathrm{r}}+n_{i}^{[\mathrm{a}]}+n_{i}^{[\mathrm{c}]}
$$


where $n_{i}^{[\mathrm{a}]} \sim \mathrm{CN}\left(0, \sigma_{\mathrm{a}}^{2}\right)$ and $n_{i}^{[\mathrm{c}]} \sim \mathrm{CN}\left(0, \sigma_{\mathrm{c}}^{2}\right)$ are the AWGN due to the antenna and that due to RF-band-to-baseband signal conversion, respectively. Assuming that $\left\{h_{i, m}\right\}_{m=1}^{M}$ is known at $\mathrm{T}_{i}$, an estimate of $x_{\mathrm{r}}$ is obtained as:

$$
\begin{aligned}
\hat{x}_{\mathrm{r}} & =\frac{y_{i}}{\sum_{m=1}^{M} h_{i, m}} \\
& =\sqrt{P_{\mathrm{r}}} x_{\mathrm{r}}+\frac{n_{i}^{[\mathrm{a}]}+n_{i}^{[\mathrm{c}]}}{\sum_{m=1}^{M} h_{i, m}}
\end{aligned}
$$

(For realizing this assumption, interested readers may consult [24,25]).

Denoting by $\gamma_{\mathrm{r} i}$ the instantaneous SNR of $\hat{x}_{\mathrm{r}}$ at $\mathrm{T}_{i}$, it can be shown that:

$$
\begin{aligned}
\gamma_{\mathrm{r} i} & =\frac{P_{\mathrm{r}}\left|\sum_{m=1}^{M} h_{i, m}\right|^{2}}{\sigma_{\mathrm{a}}^{2}+\sigma_{\mathrm{c}}^{2}} \\
& =\frac{\eta \rho M\left(P \sigma^{2}+\sigma_{\mathrm{a}}^{2}\right)\left|\sum_{m=1}^{M} h_{i, m}\right|^{2}}{\sigma_{\mathrm{a}}^{2}+\sigma_{\mathrm{c}}^{2}} .
\end{aligned}
$$

Defining $\beta:=\left|\sum_{m=1}^{M} h_{i, m}\right|^{2}$ and using (p. 48, [27]) and (Equations (5)-(8), [28]), the PDF of $\beta$ can be expressed as:

$$
f_{\beta}(x)=\frac{1}{M \sigma^{2}} \exp \left(-\frac{x}{M \sigma^{2}}\right) ; x \geq 0 .
$$

From Equations (13) and (14), we have:

$$
f_{\gamma_{\mathrm{ri}}}(x)=\frac{\sigma_{\mathrm{a}}^{2}+\sigma_{\mathrm{c}}^{2}}{\eta \rho M^{2} \sigma^{2}\left(P \sigma^{2}+\sigma_{\mathrm{a}}^{2}\right)} \exp \left(-\frac{\left(\sigma_{\mathrm{a}}^{2}+\sigma_{\mathrm{c}}^{2}\right)}{\eta \rho M^{2} \sigma^{2}\left(P \sigma^{2}+\sigma_{\mathrm{a}}^{2}\right)} x\right) ; x \geq 0 .
$$

Following the same procedure as in (9), the average BER associated with $\gamma_{\mathrm{ri}}$ is obtained as:

$$
\begin{aligned}
\bar{P}_{\mathrm{e}, \mathrm{ri}}= & \frac{M \sigma\left(\sigma_{\mathrm{a}}^{2}+\sigma_{\mathrm{c}}^{2}\right)\left[\eta \rho\left(P \sigma^{2}+\sigma_{\mathrm{a}}^{2}\right)\right]^{1 / 2}}{4\left[\eta \rho M^{2} \sigma^{2}\left(P \sigma^{2}+\sigma_{\mathrm{a}}^{2}\right)+\sigma_{\mathrm{a}}^{2}+\sigma_{\mathrm{c}}^{2}\right]^{3 / 2}} \\
& \times_{2} \mathrm{~F}_{1}\left(1, \frac{3}{2} ; 2 ; \frac{\sigma_{\mathrm{a}}^{2}+\sigma_{\mathrm{c}}^{2}}{\eta \rho M^{2} \sigma^{2}\left(P \sigma^{2}+\sigma_{\mathrm{a}}^{2}\right)+\sigma_{\mathrm{a}}^{2}+\sigma_{\mathrm{c}}^{2}}\right) .
\end{aligned}
$$

At the end node $\mathrm{T}_{i}$, the intended signal $x_{j}(j=1,2 ; j \neq i)$ can be finally recovered by performing bit-level network decoding of $\hat{x}_{\mathrm{r}}$ with its own signal $x_{i}$, and the corresponding end-to-end BER can be obtained as:

$$
\begin{aligned}
\bar{P}_{\mathrm{e}} & =\bar{P}_{\mathrm{e}, \mathrm{XOR}}\left(1-\bar{P}_{\mathrm{e}, \mathrm{r} i}\right)+\bar{P}_{\mathrm{e}, \mathrm{ri}}\left(1-\bar{P}_{\mathrm{e}, \mathrm{XOR}}\right) \\
& =\bar{P}_{\mathrm{e}, \mathrm{XOR}}+\bar{P}_{\mathrm{e}, \mathrm{r} i}-2 \bar{P}_{\mathrm{e}, \mathrm{XOR}} \bar{P}_{\mathrm{e}, \mathrm{r} i}
\end{aligned}
$$

where:

$$
\begin{aligned}
\bar{P}_{\mathrm{e}, \mathrm{XOR}} & =\bar{P}_{\mathrm{e}, 1 \mathrm{r}}\left(1-\bar{P}_{\mathrm{e}, 2 \mathrm{r}}\right)+\bar{P}_{\mathrm{e}, 2 \mathrm{r}}\left(1-\bar{P}_{\mathrm{e}, 1 \mathrm{r}}\right) \\
& =\bar{P}_{\mathrm{e}, 1 \mathrm{r}}+\bar{P}_{\mathrm{e}, 2 \mathrm{r}}-2 \bar{P}_{\mathrm{e}, 1 \mathrm{r}} \overline{\mathrm{P}}_{\mathrm{e}, 2 \mathrm{r}}
\end{aligned}
$$

is the average BER of $\hat{b}_{1} \oplus \hat{b}_{2}$.

\subsection{PS-DF-STC Scheme}

For the MA phase, the description of the signal transmissions from the end nodes $T_{1}$ and $T_{2}$ to the relay node R can be done as in the PS-DF scheme, i.e., Equations (2)-(4). The aforementioned ZF estimation and bit-level NC also follow. The corresponding average BER (i.e., $\bar{P}_{\mathrm{e}, \text { ir }}$ where $i=1,2$ ) is therefore the same as Equation (9). However, instead of transmitting the same composite bit sequence $b_{\mathrm{r}}:=\hat{b}_{1} \oplus \hat{b}_{2}$ simultaneously via $M$ antennas in the BC phase, the relay node performs space-time block coding [21] for this sequence, as outlined in [29]. Specifically, let $\mathbf{B}$ be the space-time block-coded 
composite bit matrix whose dimension is $M \times L$, where $L$ is the block length of the corresponding space-time block code. If $N$ consecutive composite bits, i.e., $b_{\mathrm{r}}[k], b_{\mathrm{r}}[k+1], \ldots, b_{\mathrm{r}}[k+N-1]$, are transmitted with this matrix, then the code rate is $N / L$. In this paper, we concentrate on the space-time block-coded composite bit matrices with the full code rate, i.e., $L=N$. Such matrices for two, three, and four antennas are shown in Table 1.

Table 1. Examples of $\mathbf{B}$ and $\mathbf{H}$ for space-time coding.

\begin{tabular}{|c|c|c|c|c|c|c|c|c|c|}
\hline$M$ & $N$ & \multicolumn{4}{|c|}{ B } & \multicolumn{4}{|c|}{$\mathbf{H}$} \\
\hline 2 & 2 & & $\begin{array}{c}b_{\mathrm{r}}[k] \\
b_{\mathrm{r}}[k+1]\end{array}$ & $\begin{array}{c}-b_{\mathrm{r}}[k+1] \\
b_{\mathrm{r}}[k]\end{array}$ & & & {$\left[\begin{array}{l}h_{i, 1} \\
h_{i, 2}\end{array}\right.$} & $\begin{array}{c}h_{i, 2} \\
-h_{i, 1}\end{array}$ & \\
\hline 3 & 4 & $\begin{array}{c}b_{\mathrm{r}}[k] \\
b_{\mathrm{r}}[k+1] \\
b_{\mathrm{r}}[k+2]\end{array}$ & $\begin{array}{c}-b_{\mathrm{r}}[k+1] \\
b_{\mathrm{r}}[k] \\
-b_{\mathrm{r}}[k+3]\end{array}$ & $\begin{array}{c}-b_{\mathrm{r}}[k+2] \\
b_{\mathrm{r}}[k+3] \\
b_{\mathrm{r}}[k]\end{array}$ & $\begin{array}{c}-b_{\mathrm{r}}[k+3] \\
-b_{\mathrm{r}}[k+2] \\
b_{\mathrm{r}}[k+1]\end{array}$ & $\begin{array}{c}h_{i, 1} \\
h_{i, 2} \\
h_{i, 3} \\
0\end{array}$ & $\begin{array}{c}h_{i, 2} \\
-h_{i, 1} \\
0 \\
-h_{i, 3}\end{array}$ & $\begin{array}{c}h_{i, 3} \\
0 \\
-h_{i, 1} \\
h_{i, 2}\end{array}$ & $\begin{array}{c}0 \\
h_{i, 3} \\
-h_{i, 2} \\
-h_{i, 1}\end{array}$ \\
\hline 4 & 4 & $\begin{array}{c}b_{\mathrm{r}}[k] \\
b_{\mathrm{r}}[k+1] \\
b_{\mathrm{r}}[k+2] \\
b_{\mathrm{r}}[k+3]\end{array}$ & $\begin{array}{c}-b_{\mathrm{r}}[k+1] \\
b_{\mathrm{r}}[k] \\
-b_{\mathrm{r}}[k+3] \\
b_{\mathrm{r}}[k+2]\end{array}$ & $\begin{array}{c}-b_{\mathrm{r}}[k+2] \\
b_{\mathrm{r}}[k+3] \\
b_{\mathrm{r}}[k] \\
-b_{\mathrm{r}}[k+1]\end{array}$ & $\begin{array}{c}-b_{\mathrm{r}}[k+3] \\
-b_{\mathrm{r}}[k+2] \\
b_{\mathrm{r}}[k+1] \\
b_{\mathrm{r}}[k]\end{array}$ & $\begin{array}{l}h_{i, 1} \\
h_{i, 2} \\
h_{i, 3} \\
h_{i, 4}\end{array}$ & $\begin{array}{c}h_{i, 2} \\
-h_{i, 1} \\
h_{i, 4} \\
-h_{i, 3}\end{array}$ & $\begin{array}{c}h_{i, 3} \\
-h_{i, 4} \\
-h_{i, 1} \\
h_{i, 2}\end{array}$ & $\begin{array}{c}h_{i, 4} \\
h_{i, 3} \\
-h_{i, 2} \\
-h_{i, 1}\end{array}$ \\
\hline
\end{tabular}

As a result, the sampled received (baseband) signal at the end node $\mathrm{T}_{i}(i=1,2)$ in the $\mathrm{BC}$ phase can be expressed as:

$$
\begin{aligned}
& {\left[\begin{array}{llll}
y_{i}[k] & y_{i}[k+1] & \cdots & y_{i}[k+N-1]
\end{array}\right]=\sqrt{P_{\mathrm{r}}}\left[\begin{array}{llll}
x_{\mathrm{r}}[k] & x_{\mathrm{r}}[k+1] & \cdots & x_{\mathrm{r}}[k+N-1]
\end{array}\right] \mathbf{H}} \\
& +\left[\begin{array}{llll}
n_{i}[k] & n_{i}[k+1] & \cdots & n_{i}[k+N-1]
\end{array}\right]
\end{aligned}
$$

where $\mathbf{H}$ is exemplified in Table 1 , and $n_{i} \sim C N\left(0, \sigma_{\mathrm{a}}^{2}+\sigma_{\mathrm{c}}^{2}\right)$ includes the antenna and signal-conversion AWGNs at the corresponding time instants. Following [30] and assuming that $\left\{h_{i, m}\right\}_{m=1}^{M}$ are known at $\mathrm{T}_{i}$, an estimate of $\left[\begin{array}{llll}x_{\mathrm{r}}[k] & x_{\mathrm{r}}[k+1] & \cdots & x_{\mathrm{r}}[k+N-1]\end{array}\right]$ can be obtained as

$$
\left[\begin{array}{llll}
\hat{x}_{\mathrm{r}}[k] & \hat{x}_{\mathrm{r}}[k+1] & \cdots & \hat{x}_{\mathrm{r}}[k+N-1]
\end{array}\right]=\operatorname{Re}\left[\begin{array}{llll}
y_{i}[k] & y_{i}[k+1] & \cdots & y_{i}[k+N-1]
\end{array}\right] \mathbf{H}^{\mathrm{H}} .
$$

It is straightforward to show that the instantaneous SNR of $\hat{x}_{\mathrm{r}}$ at $\mathrm{T}_{i}$ is:

$$
\begin{aligned}
\gamma_{\mathrm{r} i} & =\frac{P_{\mathrm{r}} \sum_{m=1}^{M}\left|h_{i, m}\right|^{2}}{\sigma_{\mathrm{a}}^{2}+\sigma_{\mathrm{c}}^{2}} \\
= & \frac{\eta \rho M\left(P \sigma^{2}+\sigma_{\mathrm{a}}^{2}\right) \sum_{m=1}^{M}\left|h_{i, m}\right|^{2}}{\sigma_{\mathrm{a}}^{2}+\sigma_{\mathrm{c}}^{2}} .
\end{aligned}
$$

Defining $\vartheta:=\sum_{m=1}^{M}\left|h_{i, m}\right|^{2}$ and using (p. 48, [27]) and (Section "Related distributions", [31]), the PDF of $\vartheta$ can be expressed as:

$$
f_{\vartheta}(x)=\frac{x^{M-1}}{\Gamma(M) \sigma^{2 M}} \exp \left(-\frac{x}{\sigma^{2}}\right) ; x \geq 0 .
$$

From Equations (21) and (22), we have:

$$
\begin{aligned}
f_{\gamma_{\mathrm{ri}}}(x)= & \frac{\left(\sigma_{\mathrm{a}}^{2}+\sigma_{\mathrm{c}}^{2}\right)^{M}}{\Gamma(M) \sigma^{2 M}\left(\eta \rho M\left(P \sigma^{2}+\sigma_{\mathrm{a}}^{2}\right)\right)^{M}} x^{M-1} \\
& \times \exp \left(-\frac{\sigma_{a}^{2}+\sigma_{c}^{2}}{\eta \rho M \sigma^{2}\left(P \sigma^{2}+\sigma_{\mathrm{a}}^{2}\right)} x\right) ; x \geq 0 .
\end{aligned}
$$


Following the same procedure as in (9), the average BER associated with $\gamma_{\mathrm{r} i}$ is obtained as:

$$
\begin{aligned}
\bar{P}_{\mathrm{e}, \mathrm{ri}}= & \frac{\sigma \Gamma(M+1 / 2)\left(\eta \rho M\left(P \sigma^{2}+\sigma_{\mathrm{a}}^{2}\right)\right)^{1 / 2}\left(\sigma_{\mathrm{a}}^{2}+\sigma_{\mathrm{c}}^{2}\right)^{M}}{2 \sqrt{\pi} \Gamma(M+1)\left(\eta \rho M \sigma^{2}\left(P \sigma^{2}+\sigma_{\mathrm{a}}^{2}\right)+\sigma_{\mathrm{a}}^{2}+\sigma_{\mathrm{c}}^{2}\right)^{M+1 / 2}} \\
& \times_{2} \mathrm{~F}_{1}\left(1, M+\frac{1}{2} ; M+1 ; \frac{\sigma_{\mathrm{a}}^{2}+\sigma_{\mathrm{c}}^{2}}{\eta \rho M \sigma^{2}\left(P \sigma^{2}+\sigma_{\mathrm{a}}^{2}\right)+\sigma_{\mathrm{a}}^{2}+\sigma_{\mathrm{c}}^{2}}\right) .
\end{aligned}
$$

At the end node $\mathrm{T}_{i}$, the intended signal $x_{j}(j=1,2 ; j \neq i)$ can be finally recovered by performing bit-level network decoding of $\hat{x}_{\mathrm{r}}$ with its own signal $x_{i}$, and the corresponding end-to-end BER is obtained using Equation (17).

\subsection{PS-AF Scheme}

For the MA phase, the description of the signal transmissions from the end nodes $\mathrm{T}_{1}$ and $\mathrm{T}_{2}$ to the relay node R can be done as in the PS-DF scheme, i.e., Equations (2)-(4). In the BC phase, the relay node amplifies and forwards the information signal as:

$$
\mathbf{z}_{\mathrm{r}}=\frac{\widetilde{\mathbf{y}}_{\mathrm{r}}}{\left\|\widetilde{\mathbf{y}}_{\mathrm{r}}\right\|}
$$

where $\left\|\widetilde{\mathbf{y}}_{\mathrm{r}}\right\|=\sqrt{(1-\rho)\left(P_{1}\left\|\mathbf{h}_{1}\right\|^{2}+P_{2}\left\|\mathbf{h}_{2}\right\|^{2}+M \sigma_{\mathrm{a}}^{2}\right)+M \sigma_{\mathrm{c}}^{2}}$ and the sampled received (baseband) signal at the end node $\mathrm{T}_{i}(i=1,2)$ is given by:

$$
\begin{aligned}
y_{i}= & \sqrt{P_{\mathrm{r}}} \mathbf{h}_{i}^{\mathrm{T}} \frac{\widetilde{\mathbf{y}}_{\mathrm{r}}}{\left\|\tilde{\mathbf{y}}_{\mathrm{r}}\right\|}+n_{i}^{[\mathrm{a}]}+n_{i}^{[\mathrm{c}]} \\
= & \frac{\sqrt{(1-\rho) P_{\mathrm{r}} P_{1}}}{\left\|\widetilde{\mathbf{y}}_{\mathrm{r}}\right\|} \mathbf{h}_{i}^{\mathrm{T}} \mathbf{h}_{1} x_{1}+\frac{\sqrt{(1-\rho) P_{\mathrm{r}} P_{2}}}{\left\|\widetilde{\mathbf{y}}_{\mathbf{r}}\right\|} \mathbf{h}_{i}^{\mathrm{T}} \mathbf{h}_{2} x_{2}+\frac{\sqrt{(1-\rho) P_{r}}}{\left\|\widetilde{\mathbf{y}}_{\mathrm{r}}\right\|} \mathbf{h}_{i}^{\mathrm{T}} \mathbf{n}_{\mathrm{r}}^{[\mathrm{a}]} \\
& +\frac{\sqrt{P_{r}}}{\left\|\widetilde{\mathbf{y}}_{\mathrm{r}}\right\|} \mathbf{h}_{i}^{\mathrm{T}} \mathbf{n}_{\mathrm{r}}^{[\mathrm{c}]}+n_{i}^{\mathrm{a}]}+n_{i}^{[\mathrm{c}]}
\end{aligned}
$$

where $n_{i}^{[\mathrm{a}]}$ and $n_{i}^{[\mathrm{c}]}$ are defined below Equation (11). Assuming that $\rho, P_{\mathrm{r}},\left\{h_{1, m}\right\}_{m=1}^{M},\left\{h_{2, m}\right\}_{m=1}^{M}$, and $\left\|\widetilde{\mathbf{y}}_{\mathrm{r}}\right\|$ are known at $\mathrm{T}_{i}$, an estimate of the intended signal $x_{j}(j=1,2 ; j \neq i)$ can be obtained as:

$$
\hat{x}_{j}=\frac{y_{i}-\sqrt{(1-\rho) P_{\mathrm{r}} P_{i}} \mathbf{h}_{i}^{\mathrm{T}} \mathbf{h}_{i} x_{i} /\left\|\widetilde{\mathbf{y}}_{\mathrm{r}}\right\|}{\sqrt{(1-\rho) P_{\mathrm{r}}} \mathbf{h}_{i}^{\mathrm{T}} \mathbf{h}_{j} /\left\|\widetilde{\mathbf{y}}_{\mathrm{r}}\right\|} .
$$

Let $\gamma_{j i}$ denote the instantaneous SNR of $\hat{x}_{j}$ at $\mathrm{T}_{i}$. It is straightforward to show that:

$$
\gamma_{j i}=\frac{(1-\rho) P_{\mathrm{r}} P_{j}\left|\sum_{m=1}^{M} h_{i, m} h_{j, m}\right|^{2}}{\left((1-\rho) \sigma_{\mathrm{a}}^{2}+\sigma_{\mathrm{c}}^{2}\right) P_{\mathrm{r}} \sum_{m=1}^{M}\left|h_{i, m}\right|^{2}+\left(\sigma_{\mathrm{a}}^{2}+\sigma_{\mathrm{c}}^{2}\right)\left[(1-\rho)\left(P_{1} \sum_{m=1}^{M}\left|h_{1, m}\right|^{2}+P_{2} \sum_{m=1}^{M}\left|h_{2, m}\right|^{2}+M \sigma_{\mathrm{a}}^{2}\right)+M \sigma_{\mathrm{c}}^{2}\right]}
$$

and the corresponding end-to-end BER, $\bar{P}_{\mathrm{e}}$, is the average BER associated with $\gamma_{j i}$. Unfortunately, it is difficult, if not impossible, to find the PDF of $\gamma_{j i}$. Therefore, the end-to-end BER has no closed-form expression and is obtained by means of simulations.

\section{TSR Protocol}

Figure 3 illustrates the key parameters in the TSR protocol for energy harvesting and information processing at the relay node $\mathrm{R}$ and the block diagram of the corresponding receiver. In Figure $3 a, \alpha$ is the fraction of the total block time $2 N$ in which the relay node harvests energy from the end nodes $\mathrm{T}_{1}$ and $\mathrm{T}_{2}$, where $0 \leq \alpha \leq 1$. The remaining block time $2(1-\alpha) N$ is used for information transmission, such that the first half of that is used for MA and the second half is used for BC. Assuming that the TSR protocol has the same energy constraint as the PSR protocol, the power of the total received signal at 
the relay node (during energy harvesting time and the MA phase) is $P^{\prime}=P /(1+\alpha)$. In what follows, we describe three multiple-antenna relaying schemes, which correspond to the TSR protocol.

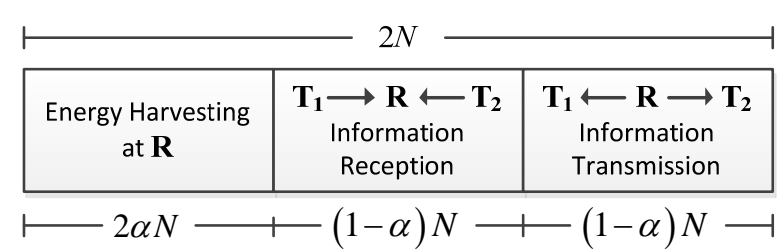

(a)

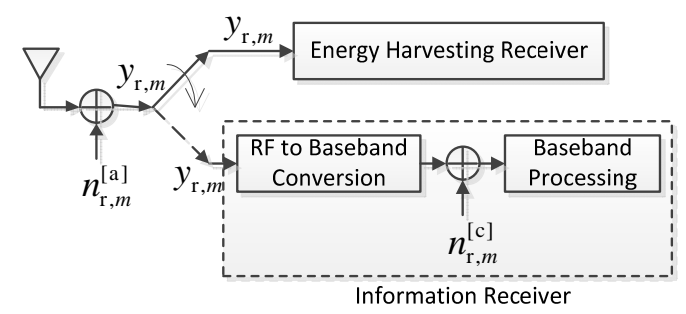

(b)

Figure 3. (a) Key parameters in the TSR protocol for energy harvesting and information processing at the relay node; and (b) Block diagram of the relay receiver (with a focus on its $m$-th antenna) in the TSR protocol.

\subsection{TS-DF Scheme}

In the MA phase, the received RF signal at the relay node $R$ is the same as Equation (2) except that $P_{1}=\zeta_{1} P^{\prime}, P_{2}=\zeta_{2} P^{\prime}$, and the harvested energy during the energy harvesting time is given by:

$$
E_{m}=\frac{2 \alpha \eta N\left(P \sigma^{2}+(1+\alpha) \sigma_{\mathrm{a}}^{2}\right)}{1+\alpha}, m=1,2, \ldots, M .
$$

After RF-band-to-baseband signal conversion at the information receiver as shown in Figure 3b, the sampled baseband signal vector is given by:

$$
\begin{aligned}
& \widetilde{\mathbf{y}}_{\mathrm{r}}=\left[\begin{array}{c}
\sqrt{P_{1}} h_{1,1} x_{1}+\sqrt{P_{2}} h_{2,1} x_{1}+n_{\mathrm{r}, 1}^{[\mathrm{a}]}+n_{\mathrm{r}, 1}^{[\mathrm{c}]} \\
\sqrt{P_{1}} h_{1,2} x_{1}+\sqrt{P_{2}} h_{2,2} x_{2}+n_{\mathrm{r}, 2}^{\mathrm{a}]}+n_{\mathrm{r}, 2}^{\mathrm{c}]} \\
\vdots \\
\sqrt{P_{1}} h_{1, M} x_{1}+\sqrt{P_{2}} h_{2, M} x_{2}+n_{\mathrm{r}, M}^{[\mathrm{a}]}+n_{\mathrm{r}, M}^{[\mathrm{c}]}
\end{array}\right] \\
& =\left[\begin{array}{cc}
\sqrt{P_{1}} h_{1,1} & \sqrt{P_{2}} h_{2,1} \\
\sqrt{P_{1}} h_{1,2} & \sqrt{P_{2}} h_{2,2} \\
\vdots & \vdots \\
\sqrt{P_{1}} h_{1, M} & \sqrt{P_{2}} h_{2, M}
\end{array}\right]\left[\begin{array}{l}
x_{1} \\
x_{2}
\end{array}\right]+\left[\begin{array}{c}
n_{\mathrm{r}, 1}^{[\mathrm{a}]} \\
n_{\mathrm{r}, 2}^{[\mathrm{a}]} \\
\vdots \\
n_{\mathrm{r}, M}^{[\mathrm{a}]}
\end{array}\right]+\left[\begin{array}{c}
n_{\mathrm{r}, 1}^{[\mathrm{c}]} \\
n_{\mathrm{r}, 2}^{[\mathrm{c}]} \\
\vdots \\
n_{\mathrm{r}, M}^{[\mathrm{c}]}
\end{array}\right] \\
& =\left[\begin{array}{ll}
\sqrt{P_{1}} \mathbf{h}_{1} & \sqrt{P_{2}} \mathbf{h}_{2}
\end{array}\right]\left[\begin{array}{l}
x_{1} \\
x_{2}
\end{array}\right]+\left(\mathbf{n}_{\mathrm{r}}^{[\mathrm{a}]}+\mathbf{n}_{\mathrm{r}}^{[\mathrm{c}]}\right) \\
& =\Xi\left[\begin{array}{l}
x_{1} \\
x_{2}
\end{array}\right]+\mathbf{v}_{\mathbf{r}}
\end{aligned}
$$

where $n_{\mathrm{r}, m}^{[\mathrm{c}]} \sim C N\left(0, \sigma_{\mathrm{c}}^{2}\right)$ is the AWGN due to such conversion. Assuming that $P_{1}, P_{2},\left\{h_{1, m}\right\}_{m=1}^{M}$, and $\left\{h_{2, m}\right\}_{m=1}^{M}$ are known at the relay node and applying ZF detection, estimates of the information signals $x_{1}$ and $x_{2}$, denoted by $\hat{x}_{1}$ and $\hat{x}_{2}$. respectively, are obtained as:

$$
\left[\begin{array}{l}
\hat{x}_{1} \\
\hat{x}_{2}
\end{array}\right]=\left(\Xi^{\mathrm{H}} \boldsymbol{\Xi}\right)^{-1} \Xi \widetilde{\mathbf{y}}_{\mathrm{r}} .
$$


It is straightforward to show that the instantaneous SNR of $\hat{x}_{i}$ is:

$$
\begin{aligned}
\gamma_{i \mathrm{r}} & =\frac{1}{(1+\alpha)\left(\sigma_{\mathrm{a}}^{2}+\sigma_{\mathrm{c}}^{2}\right)\left[\left(\Xi^{\mathrm{H}} \Xi\right)^{-1}\right]_{i i}} \\
& =\frac{\zeta_{i} P \sum_{k=1}^{M-1} \sum_{l=k+1}^{M}\left|h_{1, k} h_{2, l}-h_{1, l} h_{2, k}\right|^{2}}{(1+\alpha)\left(\sigma_{\mathrm{a}}^{2}+\sigma_{\mathrm{c}}^{2}\right) \sum_{m=1}^{M}\left|h_{j, m}\right|^{2}}
\end{aligned}
$$

where $i=1,2$. From Equations (7) and (32), we have:

$$
f_{\gamma_{i \mathrm{r}}}(x)=\frac{\left((1+\alpha)\left(\sigma_{\mathrm{a}}^{2}+\sigma_{\mathrm{c}}^{2}\right)\right)^{M-1}}{\Gamma(M-1) \sigma^{2 M-2}\left(\zeta_{i} P\right)^{M-1}} x^{M-2} \exp \left(-\frac{(1+\alpha)\left(\sigma_{\mathrm{a}}^{2}+\sigma_{\mathrm{c}}^{2}\right)}{\zeta_{i} P \sigma^{2}} x\right) ; x \geq 0 .
$$

Following the same procedure as in Equation (9), the average BER associated with $\gamma_{i r}$ is obtained as:

$$
\begin{aligned}
\bar{P}_{\mathrm{e}, \mathrm{i}}= & \frac{\sigma \Gamma(M-1 / 2)\left(\zeta_{i} P\right)^{1 / 2}\left((1+\alpha)\left(\sigma_{\mathrm{a}}^{2}+\sigma_{\mathrm{c}}^{2}\right)\right)^{M-1}}{2 \sqrt{\pi} \Gamma(M)\left(\zeta_{i} P \sigma^{2}+(1+\alpha)\left(\sigma_{\mathrm{a}}^{2}+\sigma_{\mathrm{c}}^{2}\right)\right)^{M-1 / 2}} \\
& { }_{2} F_{1}\left(1, M-\frac{1}{2} ; M ; \frac{(1+\alpha)\left(\sigma_{\mathrm{a}}^{2}+\sigma_{\mathrm{c}}^{2}\right)}{\zeta_{i} P \sigma^{2}+(1+\alpha)\left(\sigma_{\mathrm{a}}^{2}+\sigma_{\mathrm{c}}^{2}\right)}\right) .
\end{aligned}
$$

Then, the corresponding composite signal $x_{\mathrm{r}}$ is created and broadcasted in the same way as in the PS-DF case. In the BC phase, the sampled received (baseband) signal at the end node $\mathrm{T}_{i}(i=1,2)$ is the same as Equation (11) except that:

$$
\begin{aligned}
P_{\mathrm{r}} & =\frac{\sum_{m=1}^{M} E_{m}}{(1-\alpha) N} \\
& =\frac{2 \alpha \eta M\left(P \sigma^{2}+(1+\alpha) \sigma_{\mathrm{a}}^{2}\right)}{\left(1-\alpha^{2}\right)} .
\end{aligned}
$$

Assuming that $\left\{h_{i, m}\right\}_{m=1}^{M}$ are known at $\mathrm{T}_{i}$, an estimate of $x_{\mathrm{r}}$ (i.e., $\hat{x}_{\mathrm{r}}$ ) is obtained from Equation (12) together with Equation (35).

It can be shown that the instantaneous $\mathrm{SNR}$ of $\hat{x}_{\mathrm{r}}$ at $\mathrm{T}_{i}$ is:

$$
\begin{aligned}
\gamma_{\mathrm{r} i} & =\frac{P_{\mathrm{r}}\left|\sum_{m=1}^{M} h_{i, m}\right|^{2}}{\sigma_{\mathrm{a}}^{2}+\sigma_{\mathrm{c}}^{2}} \\
& =\frac{2 \alpha \eta M\left(P \sigma^{2}+(1+\alpha) \sigma_{\mathrm{a}}^{2}\right)\left|\sum_{m=1}^{M} h_{i, m}\right|^{2}}{\left(1-\alpha^{2}\right)\left(\sigma_{\mathrm{a}}^{2}+\sigma_{\mathrm{c}}^{2}\right)} .
\end{aligned}
$$

From Equations (14) and (36), we have:

$$
\begin{aligned}
f_{\gamma_{\mathrm{ri}}}(x)= & \frac{\left(1-\alpha^{2}\right)\left(\sigma_{\mathrm{a}}^{2}+\sigma_{\mathrm{c}}^{2}\right)}{2 \alpha \eta M^{2} \sigma^{2}\left(P \sigma^{2}+(1+\alpha) \sigma_{\mathrm{a}}^{2}\right)} \\
& \times \exp \left(-\frac{\left(1-\alpha^{2}\right)\left(\sigma_{\mathrm{a}}^{2}+\sigma_{\mathrm{c}}^{2}\right)}{2 \alpha \eta M^{2} \sigma^{2}\left(P \sigma^{2}+(1+\alpha) \sigma_{\mathrm{a}}^{2}\right)} x\right) ; x \geq 0 .
\end{aligned}
$$

Following the same procedure as in Equation (9), the average BER associated with $\gamma_{\mathrm{r} i}$ is obtained as:

$$
\begin{aligned}
\bar{P}_{\mathrm{e}, \mathrm{r} i}= & \frac{M \sigma\left(1-\alpha^{2}\right)\left(\sigma_{\mathrm{a}}^{2}+\sigma_{\mathrm{c}}^{2}\right)\left(\alpha \eta\left(P \sigma^{2}+(1+\alpha) \sigma_{\mathrm{a}}^{2}\right)\right)^{1 / 2}}{2 \sqrt{2}\left(2 \alpha \eta M^{2} \sigma^{2}\left(P \sigma^{2}+(1+\alpha) \sigma_{\mathrm{a}}^{2}\right)+\left(1-\alpha^{2}\right)\left(\sigma_{\mathrm{a}}^{2}+\sigma_{\mathrm{c}}^{2}\right)\right)^{3 / 2}} \\
& \times{ }_{2} \mathrm{~F}_{1}\left(1, \frac{3}{2} ; 2 ; \frac{\left(1-\alpha^{2}\right)\left(\sigma_{\mathrm{a}}^{2}+\sigma_{\mathrm{c}}^{2}\right)}{2 \alpha \eta M^{2} \sigma^{2}\left(P \sigma^{2}+(1+\alpha) \sigma_{\mathrm{a}}^{2}\right)+\left(1-\alpha^{2}\right)\left(\sigma_{\mathrm{a}}^{2}+\sigma_{\mathrm{c}}^{2}\right)}\right) .
\end{aligned}
$$

At the end node $\mathrm{T}_{i}$, the intended signal $x_{j}(j=1,2 ; j \neq i)$ can be finally recovered by performing bit-level network decoding of $\hat{x}_{\mathrm{r}}$ with its own signal $x_{i}$, and the corresponding end-to-end BER is obtained using Equation (17). 


\subsection{TS-DF-STC Scheme}

For the MA phase, the description of the signal transmissions from the end nodes $\mathrm{T}_{1}$ and $\mathrm{T}_{2}$ to the relay node $\mathrm{R}$ can be done as in the TS-DF scheme, i.e., Equations (2), (29) and (30). The aforementioned ZF estimation and bit-level NC also follow. The corresponding average BER (i.e., $\bar{P}_{\mathrm{e}, \text { ir }}$ where $i=1,2$ ) is therefore the same as Equation (34). During the BC phase, the relay node performs full-rate space-time block coding for the resultant composite bit sequence and the end nodes perform the corresponding sequence decoding and signal recovery, as in the PS-DF-STC case.

It is straightforward to show that the instantaneous SNR of an estimate of $x_{\mathrm{r}}$ at $\mathrm{T}_{i}$ is:

$$
\begin{aligned}
\gamma_{\mathrm{r} i} & =\frac{P_{\mathrm{r}} \sum_{m=1}^{M}\left|h_{i, m}\right|^{2}}{\sigma_{\mathrm{a}}^{2}+\sigma_{\mathrm{c}}^{2}} \\
& =\frac{2 \alpha \eta M\left(P \sigma^{2}+(1+\alpha) \sigma_{\mathrm{a}}^{2}\right) \sum_{m=1}^{M}\left|h_{i, m}\right|^{2}}{\left(1-\alpha^{2}\right)\left(\sigma_{\mathrm{a}}^{2}+\sigma_{\mathrm{c}}^{2}\right)} .
\end{aligned}
$$

From Equations (22) and (39), we have:

$$
\begin{aligned}
f_{\gamma_{\mathrm{ri}}}(x)= & \frac{\left(1-\alpha^{2}\right)^{M}\left(\sigma_{\mathrm{a}}^{2}+\sigma_{\mathrm{c}}^{2}\right)^{M}}{\Gamma(M)\left(2 \alpha \eta M \sigma^{2}\right)^{M}\left[P \sigma^{2}+(1+\alpha) \sigma_{2}^{2}\right]^{M}} x^{M-1} \\
& \times \exp \left(-\frac{\left(1-\alpha^{2}\right)\left(\sigma_{\mathrm{a}}^{2}+\sigma_{\mathrm{c}}^{2}\right)}{2 \alpha \eta M \sigma^{2}\left(P \sigma^{2}+(1+\alpha) \sigma_{\mathrm{a}}^{2}\right)} x\right) ; x \geq 0 .
\end{aligned}
$$

Following the same procedure as in Equation (9), the average BER associated with $\gamma_{\mathrm{r} i}$ is obtained as:

$$
\begin{aligned}
\bar{P}_{\mathrm{e}, \mathrm{ri}}= & \frac{\sigma \Gamma(M+1 / 2)\left(\alpha \eta\left(P \sigma^{2}+(1+\alpha) \sigma_{\mathrm{a}}^{2}\right)\right)^{1 / 2}\left(\left(1-\alpha^{2}\right)\left(\sigma_{\mathrm{a}}^{2}+\sigma_{\mathrm{c}}^{2}\right)\right)^{M}}{\sqrt{2 \pi M} \Gamma(M)\left(2 \alpha \eta M \sigma^{2}\left(P \sigma^{2}+(1+\alpha) \sigma_{\mathrm{a}}^{2}\right)+\left(1-\alpha^{2}\right)\left(\sigma_{\mathrm{a}}^{2}+\sigma_{\mathrm{c}}^{2}\right)\right)^{M+1 / 2}} \\
& \times_{2} F_{1}\left(1, M+\frac{1}{2} ; M+1 ; \frac{\left(1-\alpha^{2}\right)\left(\sigma_{\mathrm{a}}^{2}+\sigma_{\mathrm{c}}^{2}\right)}{2 \alpha \eta M \sigma^{2}\left(P \sigma^{2}+(1+\alpha) \sigma_{\mathrm{a}}^{2}\right)+\left(1-\alpha^{2}\right)\left(\sigma_{\mathrm{a}}^{2}+\sigma_{\mathrm{c}}^{2}\right)}\right) .
\end{aligned}
$$

The corresponding end-to-end BER is obtained using Equation (17).

\subsection{TS-AF Scheme}

For the MA phase, the description of the signal transmissions from the end nodes $T_{1}$ and $T_{2}$ to the relay node $\mathrm{R}$ can be done as in the TS-DF scheme. In the BC phase, the relay node amplifies and forwards the information signal as expressed in Equation (25), where:

$$
\left\|\widetilde{\mathbf{y}}_{\mathrm{r}}\right\|=\sqrt{P_{1}\left\|\mathbf{h}_{1}\right\|^{2}+P_{2}\left\|\mathbf{h}_{2}\right\|^{2}+M \sigma_{\mathrm{a}}^{2}+M \sigma_{\mathrm{c}}^{2}}
$$

and the sampled received (baseband) signal at the end node $\mathrm{T}_{i}(i=1,2)$ is given by:

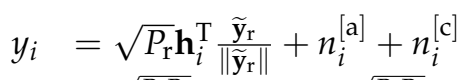

$$
\begin{aligned}
& =\frac{\sqrt{P_{\mathrm{P}} P_{1}}}{\left\|\tilde{\mathbf{y}}_{\mathbf{r}}\right\|} \mathbf{h}_{i}^{\mathrm{T}} \mathbf{h}_{1} x_{1}+\frac{\sqrt{P_{\mathrm{r}} P_{2}}}{\left\|\tilde{y}_{\mathbf{r}}\right\|} \mathbf{h}_{i}^{\mathrm{T}} \mathbf{h}_{2} x_{2}+\frac{\sqrt{P_{\mathrm{r}}}}{\left\|\tilde{\mathbf{y}}_{\mathrm{r}}\right\|} \mathbf{h}_{i}^{\mathrm{T}} \mathbf{n}_{\mathrm{r}}^{[\mathrm{a}]}+\frac{\sqrt{P_{\mathrm{r}}}}{\left\|\tilde{\mathbf{y}}_{\mathrm{r}}\right\|} \mathbf{h}_{i}^{\mathrm{T}} \mathbf{n}_{\mathrm{r}}^{[\mathrm{c}]}+n_{i}^{[\mathrm{a}]}+n_{i}^{[\mathrm{c}]}
\end{aligned}
$$

where $n_{i}^{[\mathrm{a}]}$ and $n_{i}^{[\mathrm{c}]}$ are defined below Equation (11). Assuming that $P_{\mathrm{r}},\left\{h_{1, m}\right\}_{m=1}^{M},\left\{h_{2, m}\right\}_{m=1}^{M}$, and $\left\|\widetilde{\mathbf{y}}_{\mathbf{r}}\right\|$ are known at $\mathrm{T}_{i}$, an estimate of the intended signal $x_{j}(j=1,2 ; j \neq i)$ can be obtained as:

$$
\hat{x}_{j}=\frac{y_{i}-\sqrt{P_{\mathrm{r}} P_{i}} \mathbf{h}_{i}^{\mathrm{T}} \mathbf{h}_{i} x_{i} /\left\|\widetilde{\mathbf{y}}_{\mathrm{r}}\right\|}{\sqrt{P_{\mathrm{r}}} \mathbf{h}_{i}^{\mathrm{T}} \mathbf{h}_{j} /\left\|\widetilde{\mathbf{y}}_{\mathrm{r}}\right\|}
$$


Let $\gamma_{j i}$ denote the instantaneous SNR of $\hat{x}_{j}$ at $\mathrm{T}_{i}$. It is straightforward to show that:

$$
\gamma_{j i}=\frac{P_{\mathrm{r}} P_{j}\left|\sum_{m=1}^{M} h_{i, m} h_{j, m}\right|^{2}}{\left(\sigma_{\mathrm{a}}^{2}+\sigma_{\mathrm{c}}^{2}\right)\left(P_{\mathrm{r}} \sum_{m=1}^{M}\left|h_{i, m}\right|^{2}+P_{1} \sum_{m=1}^{M}\left|h_{1, m}\right|^{2}+P_{2} \sum_{m=1}^{M}\left|h_{2, m}\right|^{2}+M \sigma_{\mathrm{a}}^{2}+M \sigma_{\mathrm{c}}^{2}\right)}
$$

and the corresponding end-to-end BER, $\bar{P}_{\mathrm{e}}$, is the average BER associated with $\gamma_{j i}$. Unfortunately, it is difficult, if not impossible, to find the PDF of $\gamma_{j i}$. Therefore, the end-to-end BER has no closed-form expression and is obtained by means of simulations.

\section{Simulation Results}

In this section, we evaluate the performance of the proposed multiple-antenna relaying schemes (i.e., PS-DF, PS-DF-STC, PS-AF, TS-DF, TS-DF-STC, and TS-AF) in terms of average BER of the end nodes $T_{1}$ and $T_{2}$. Suppose that $T_{1}$ and $T_{2}$ are separated by a distance of $2 \mathrm{~m}$, and the relay $R$ is located halfway between them. Unless stated otherwise, we set the total signal power, $P=1 \mathrm{~W}$; the power ratios of $\mathrm{T}_{1}$ and $\mathrm{T}_{2}, \zeta_{1}, \zeta_{2}=0.5$; the path loss exponent, $v=2.7$; the power energy conversion efficiency, $\eta=1$; the power splitting ratio in the PSR protocol, $\rho=0.5$; and the time fraction used for energy harvesting in the TSR protocol, $\alpha=0.5$.

The simulated BERs of the PS-DF, PS-DF-STC, and PS-AF schemes are plotted versus antenna noise variance $\sigma_{\mathrm{a}}^{2}$ for different numbers of relay antennas $M$ (with fixed conversion noise variance $\sigma_{\mathrm{c}}^{2}=0.01$ ) in Figure 4 and versus conversion noise variance $\sigma_{\mathrm{c}}^{2}$ for different values of $M$ (with fixed antenna noise variance $\left.\sigma_{\mathrm{a}}^{2}=0.01\right)$ in Figure 5. The theoretical BER curves of the PS-DF scheme (computed with Equations (9), (16), and (17)) and the PS-DF-STC scheme (computed with Equations (9), (17), and (24)) are also included and labeled with "(theor.)". We can see that the theoretical results perfectly match their simulation counterparts, which verifies the BER formulae derived in Sections 3.1 and 3.2. Similarly, the results for the TS-DF, TS-DF-STC, and TS-AF cases are shown in Figures 6 and 7. As seen, the theoretical BER results (computed with Equations (17), (34), and (38) for the TS-DF case, and with Equations (17), (34), and (41) for the TS-DF-STC case) agree well with the simulated ones, which validates the BER analysis in Sections 4.1 and 4.2. It is clear that increasing the number of relay antennas generally improves the BER performance (the results at $M=3$ are excluded to make the BER curves readable in all these figures). From Figures 4 and 5, we can see that the BERs of the PS-DF, PS-DF-STC, and PS-AF schemes are comparable when $M=2$, and their difference becomes significant when $M=4$. In the latter case, the PS-DF-STC scheme performs best while the PS-AF scheme does worst. Similar performance trends can be observed in the TS-DF, TS-DF-STC, and TS-AF schemes, as illustrated in Figures 6 and 7. Comparing Figures 4 and 6 (or Figures 5 and 7), one can find that, with the same number of relay antennas, the TS-DF and TS-AF schemes are generally superior to the PS-DF and PS-AF schemes, respectively.

It would be interesting to study the effect of the power allocation parameters, i.e., power splitting ratio $\rho$ and energy harvesting time $\alpha$, on the BER performance. To this end, we show in Figure 8 the BER as a function of $\rho$ for the PS-DF, PS-DF-STC, and PS-AF schemes, and in Figure 9 the BER as a function of $\alpha$ for the TS-DF, TS-DF-STC, and TS-AF schemes. From these two figures, we observe that, in general, equal power allocation, i.e., $\rho, \alpha=0.5$, is a good strategy for the PS-AF and TS-AF schemes. However, the optimal $\rho$ and $\alpha$ which minimize the BERs of the PS-DF and TS-DF schemes, respectively, depend mainly on the number of relay antennas $M$. Specifically, these optimal $\rho$ and $\alpha$ increase (toward 1) as $M$ increases. For example, the optimal $\rho$ for the two-antenna PS-DF scheme is approximately 0.26 while that for the four-antenna PS-DF scheme is around 0.65 (See Figure 8). In addition, the optimal $\rho$ for the two-antenna PS-DF-STC scheme (or the optimal $\alpha$ for the two-antenna TS-DF-STC scheme) is nearly the same as that for the four-antenna PS-DF-STC scheme (or that for the 
four-antenna TS-DF-STC scheme). This result indicates that these optimal $\rho$ and $\alpha$ are not sensitive to the value of $M$, unlike those for the PS-DF and TS-DF schemes, respectively.

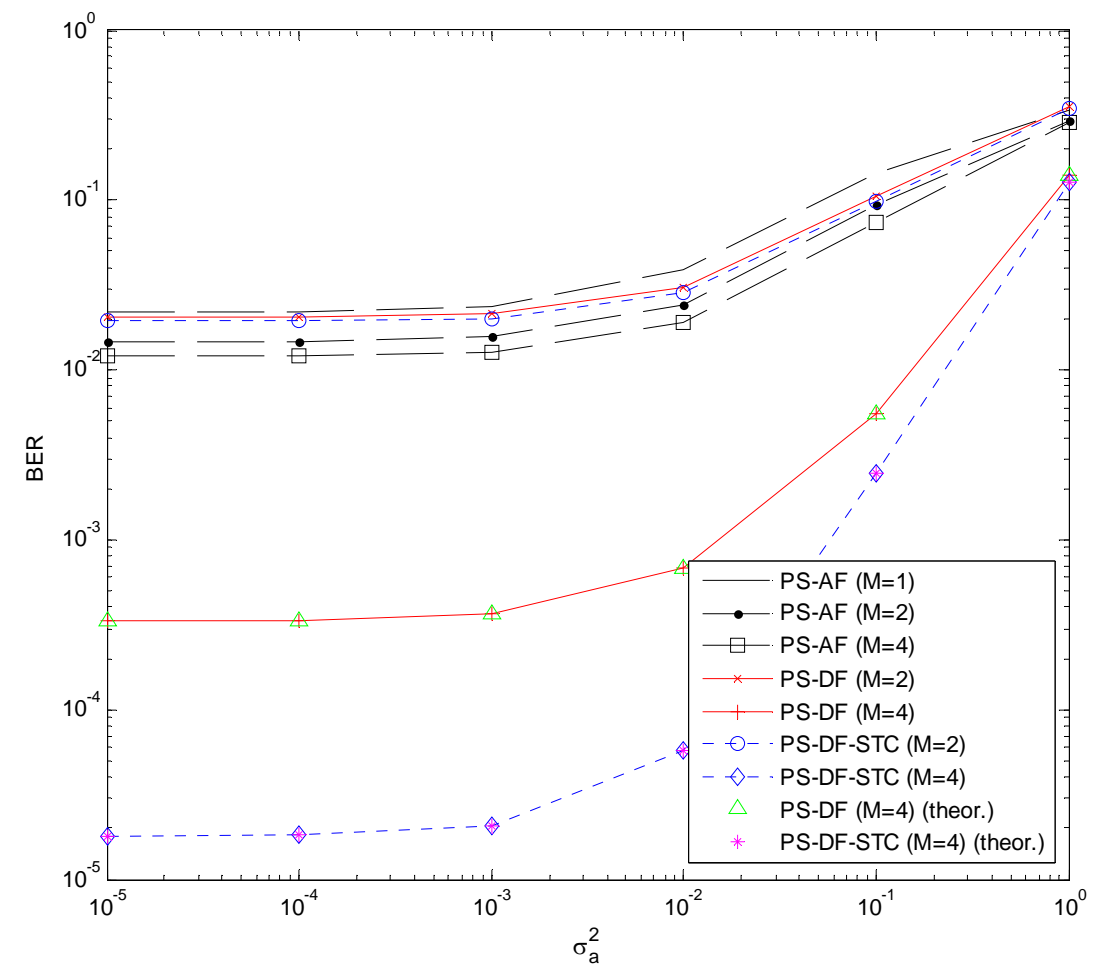

Figure 4. BER versus antenna noise variance $\sigma_{\mathrm{a}}^{2}$ for the PS-DF, PS-DF-STC, and PS-AF schemes (conversion noise variance $\sigma_{\mathrm{C}}^{2}=0.01$ and power splitting ratio $\rho=0.5$ ).

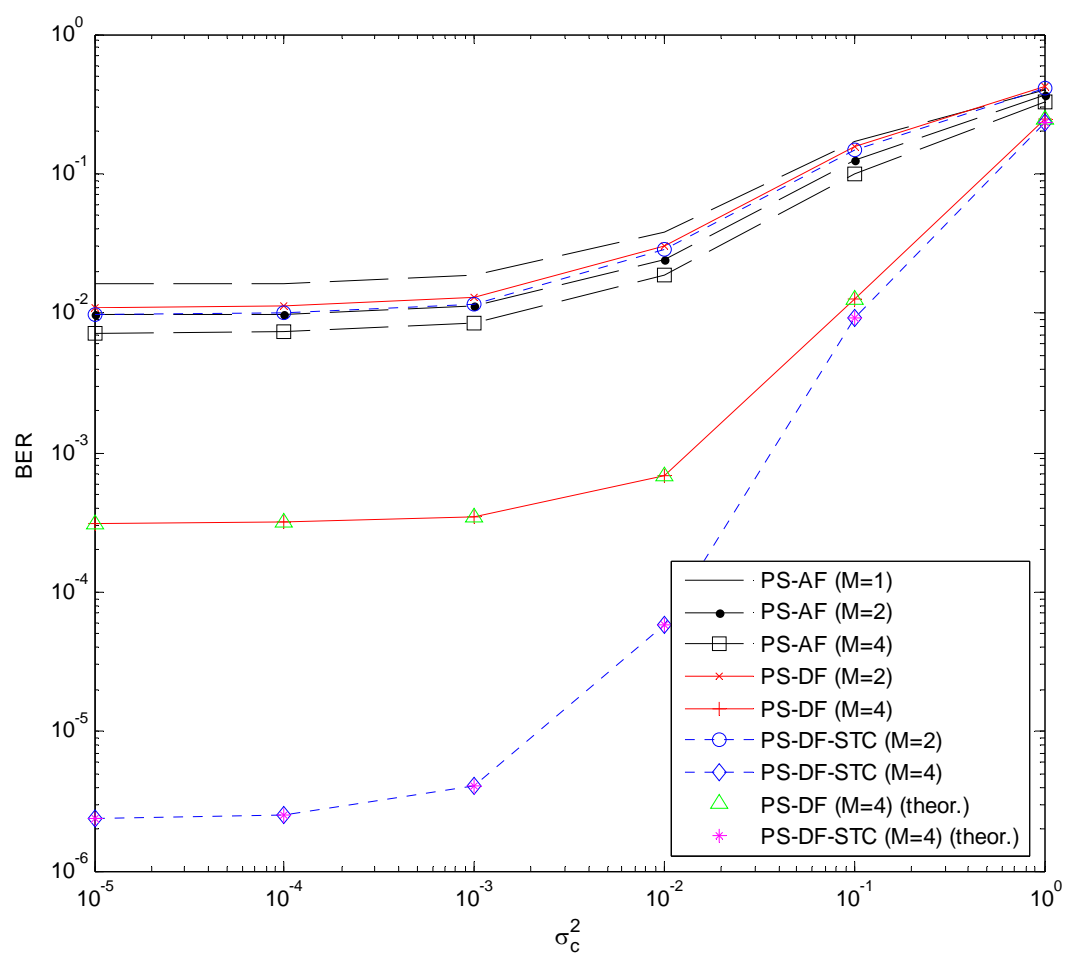

Figure 5. BER versus conversion noise variance $\sigma_{\mathrm{c}}^{2}$ for the PS-DF, PS-DF-STC, and PS-AF schemes (antenna noise variance $\sigma_{\mathrm{a}}^{2}=0.01$ and power splitting ratio $\rho=0.5$ ). 


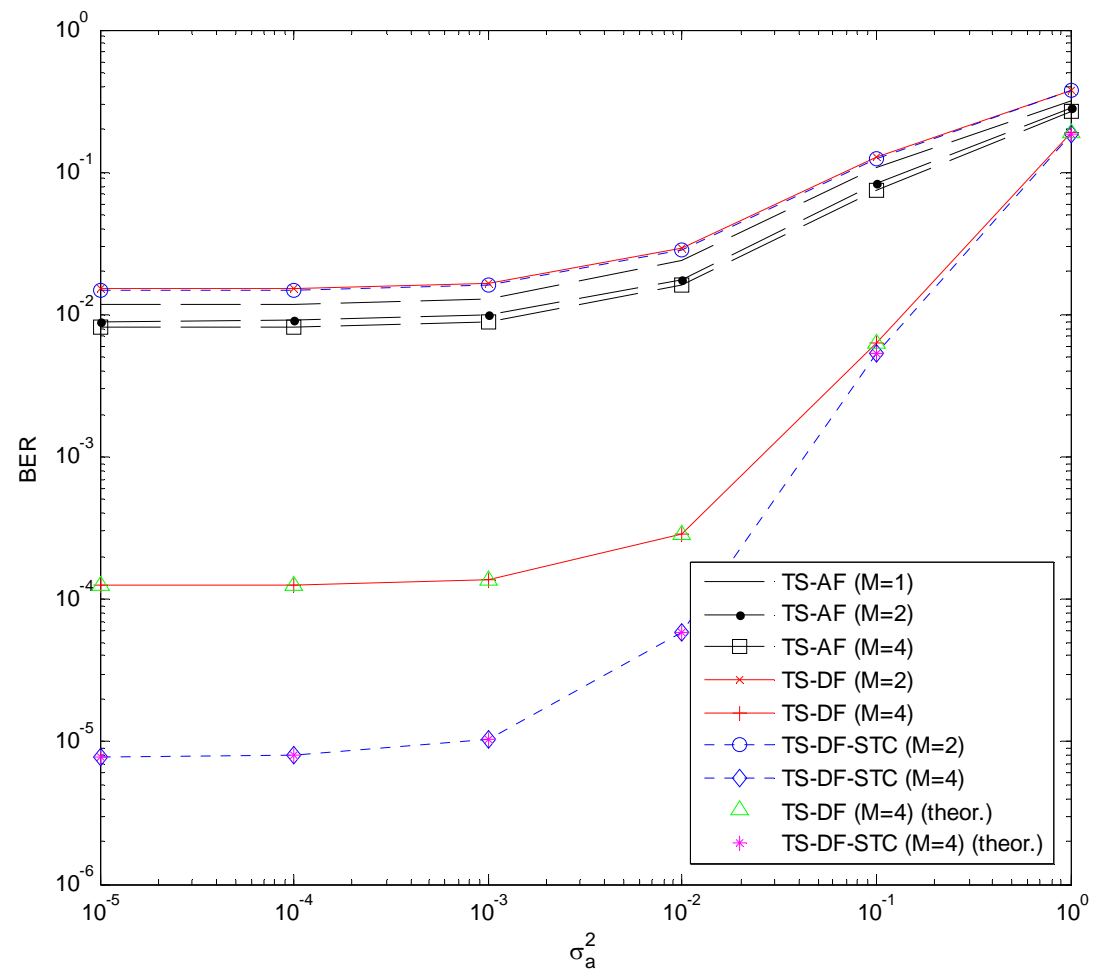

Figure 6. BER versus antenna noise variance $\sigma_{\mathrm{a}}^{2}$ for the TS-DF, TS-DF-STC, and TS-AF schemes (conversion noise variance $\sigma_{\mathrm{c}}^{2}=0.01$ and energy harvesting time $\alpha=0.5$ ).

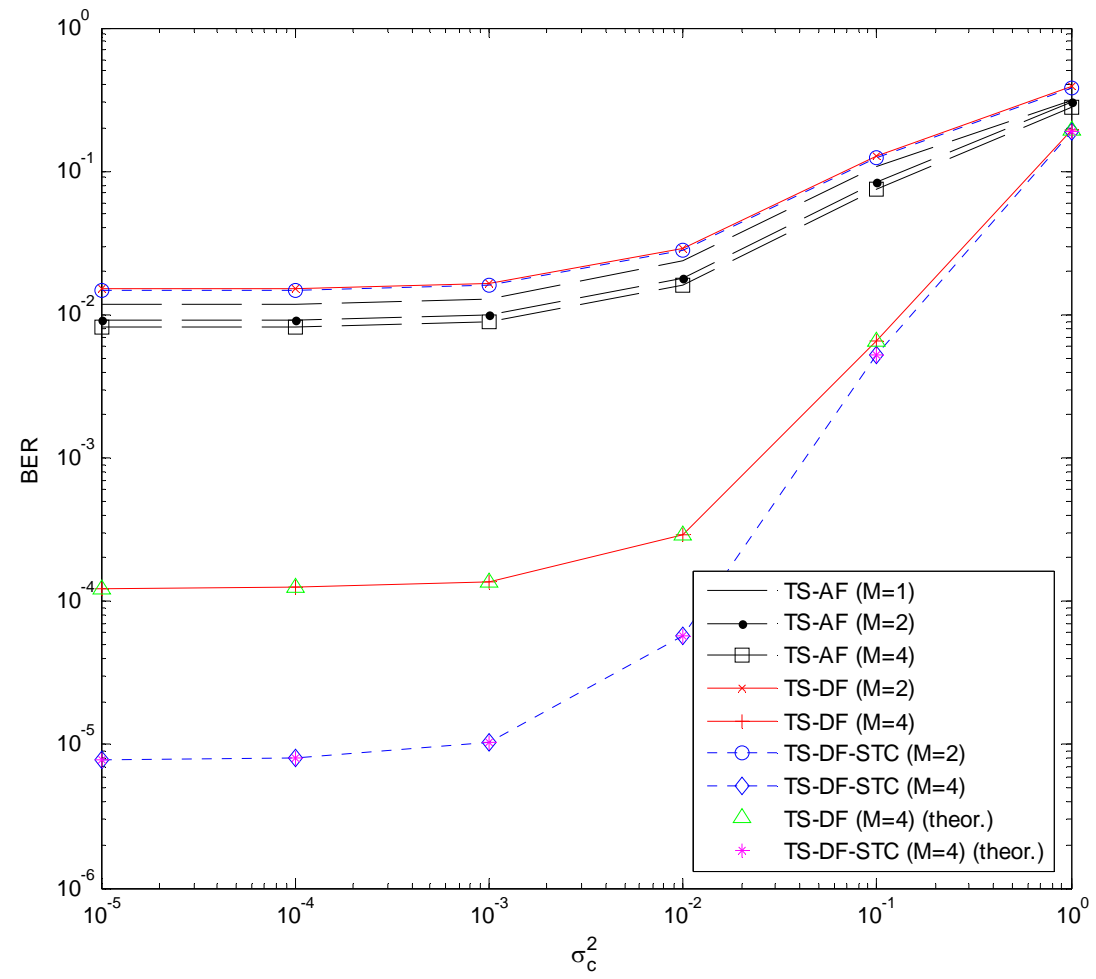

Figure 7. BER versus conversion noise variance $\sigma_{\mathrm{c}}^{2}$ for the TS-DF, TS-DF-STC, and TS-AF schemes (antenna noise variance $\sigma_{\mathrm{a}}^{2}=0.01$ and energy harvesting time $\alpha=0.5$ ). 


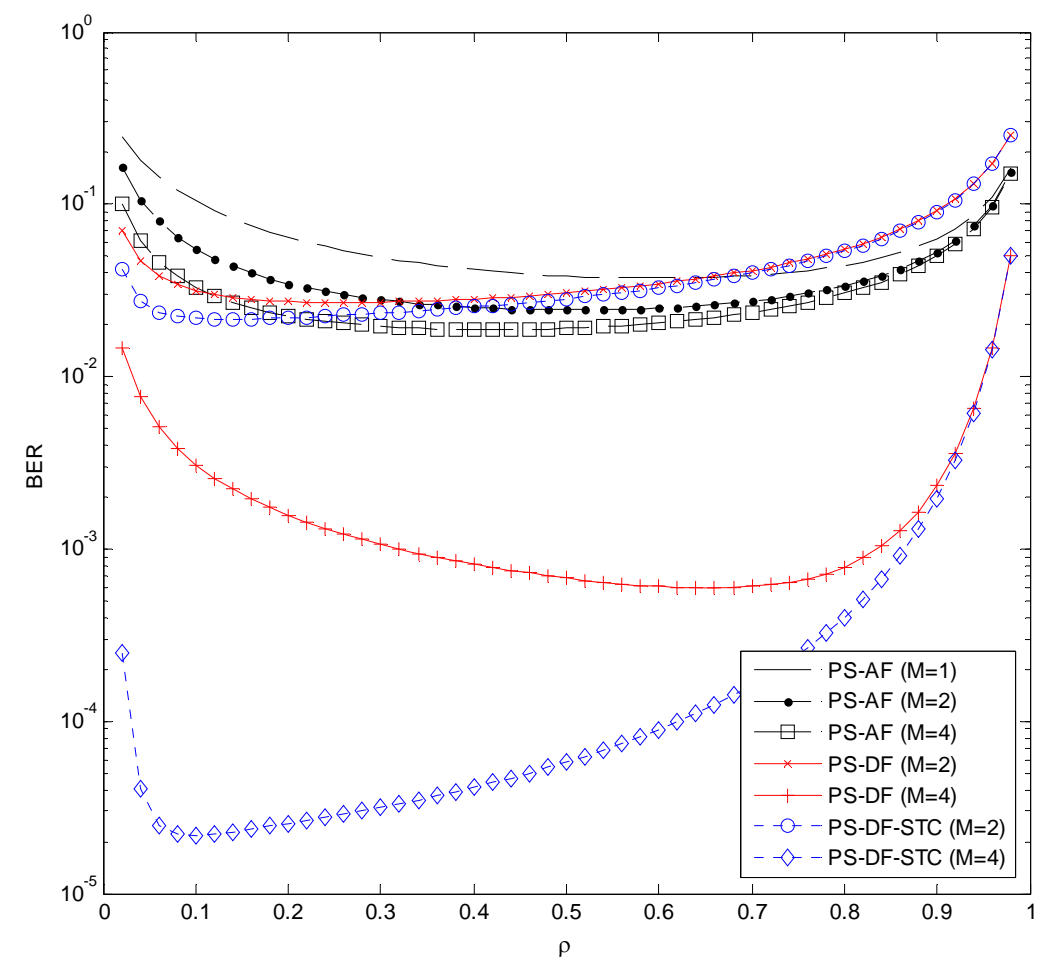

Figure 8. BER versus power splitting ratio $\rho$ for the PS-DF, PS-DF-STC, and PS-AF schemes (antenna noise variance $\sigma_{\mathrm{a}}^{2}=0.01$ and conversion noise variance $\sigma_{\mathrm{c}}^{2}=0.01$ ).

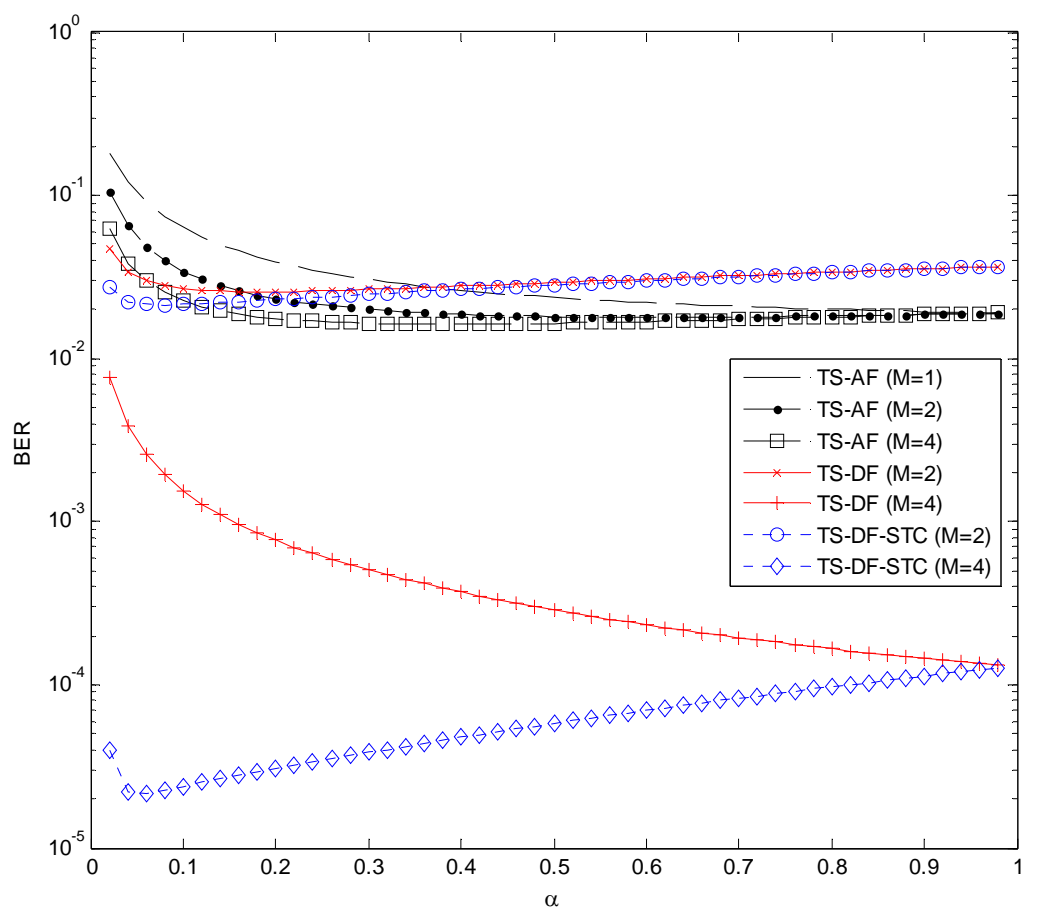

Figure 9. BER versus energy harvesting time $\alpha$ for the TS-DF, TS-DF-STC, and TS-AF schemes (antenna noise variance $\sigma_{\mathrm{a}}^{2}=0.01$ and conversion noise variance $\sigma_{\mathrm{c}}^{2}=0.01$ ).

To get a further insight into the impact of $M, \rho$, and $\alpha$, we plot the BER curves of the multiple-antenna relaying schemes with optimal power allocation (i.e., using the optimal $\rho$ or $\alpha$ ) in Figures 10-13. Comparing these results to those in Figures 4-7, we find that for all considered 
relaying schemes except TS-AF scheme, the performance gain of the optimal power allocation over the equal power allocation can become significant as $M$ increases. Similar to the case of equal power allocation, the power optimization-based TS-DF and TS-AF schemes generally outperform their PS-DF and PS-AF counterparts, respectively.

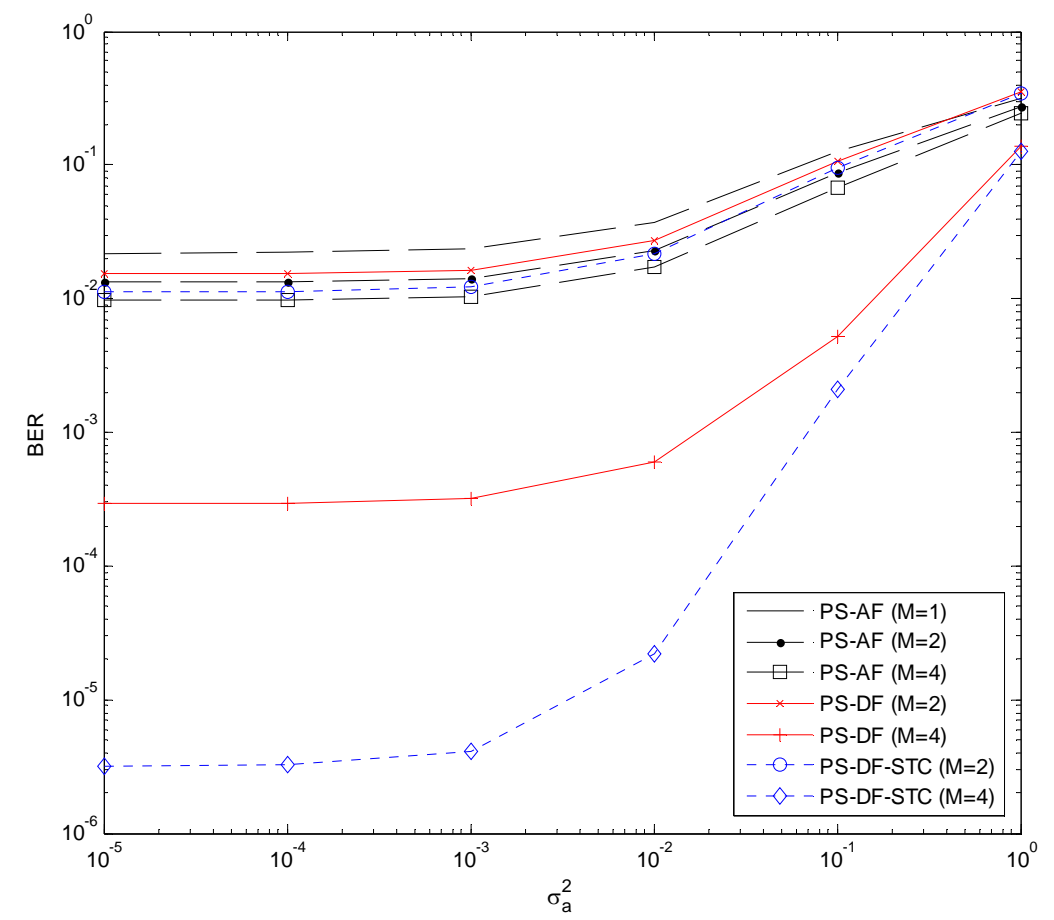

Figure 10. BER versus antenna noise variance $\sigma_{\mathrm{a}}^{2}$ for the PS-DF, PS-DF-STC, and PS-AF schemes (conversion noise variance $\sigma_{\mathrm{c}}^{2}=0.01$ and optimal power splitting ratio).

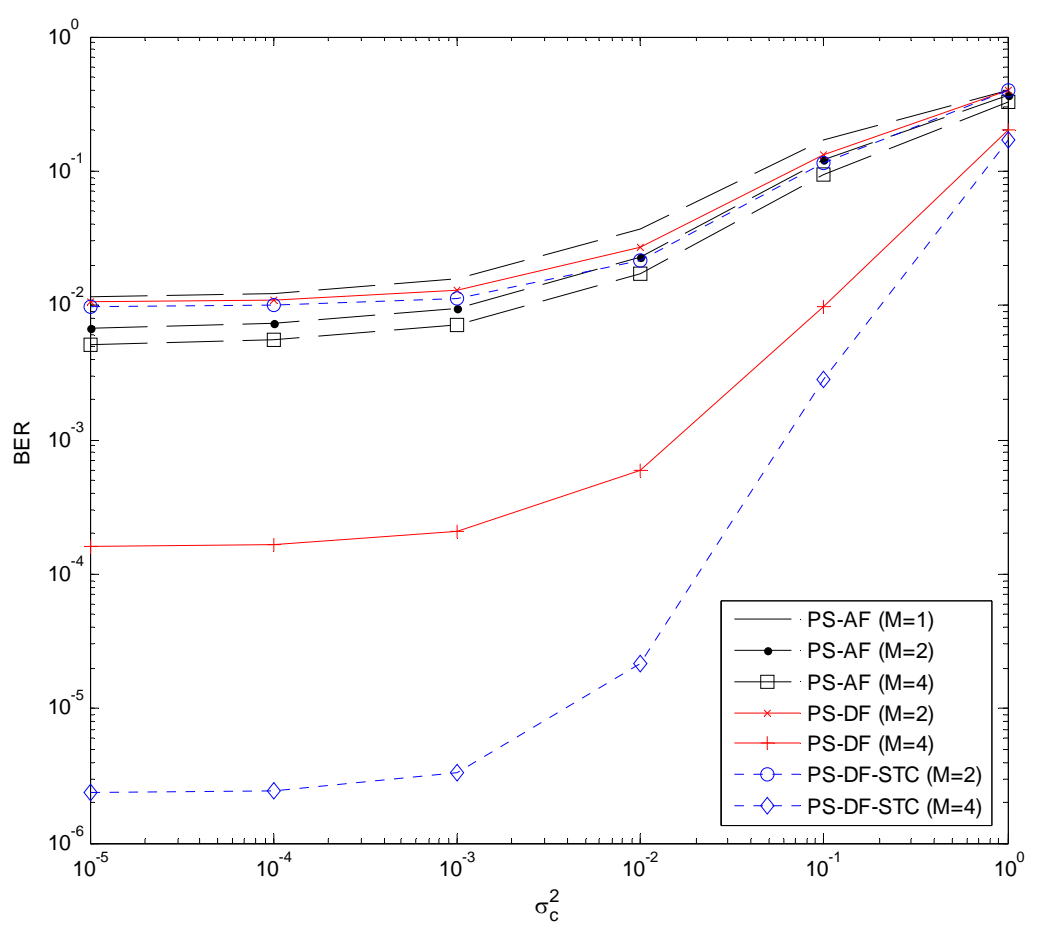

Figure 11. BER versus conversion noise variance $\sigma_{\mathrm{c}}^{2}$ for the PS-DF, PS-DF-STC, and PS-AF schemes (antenna noise variance $\sigma_{\mathrm{a}}^{2}=0.01$ and optimal power splitting ratio). 


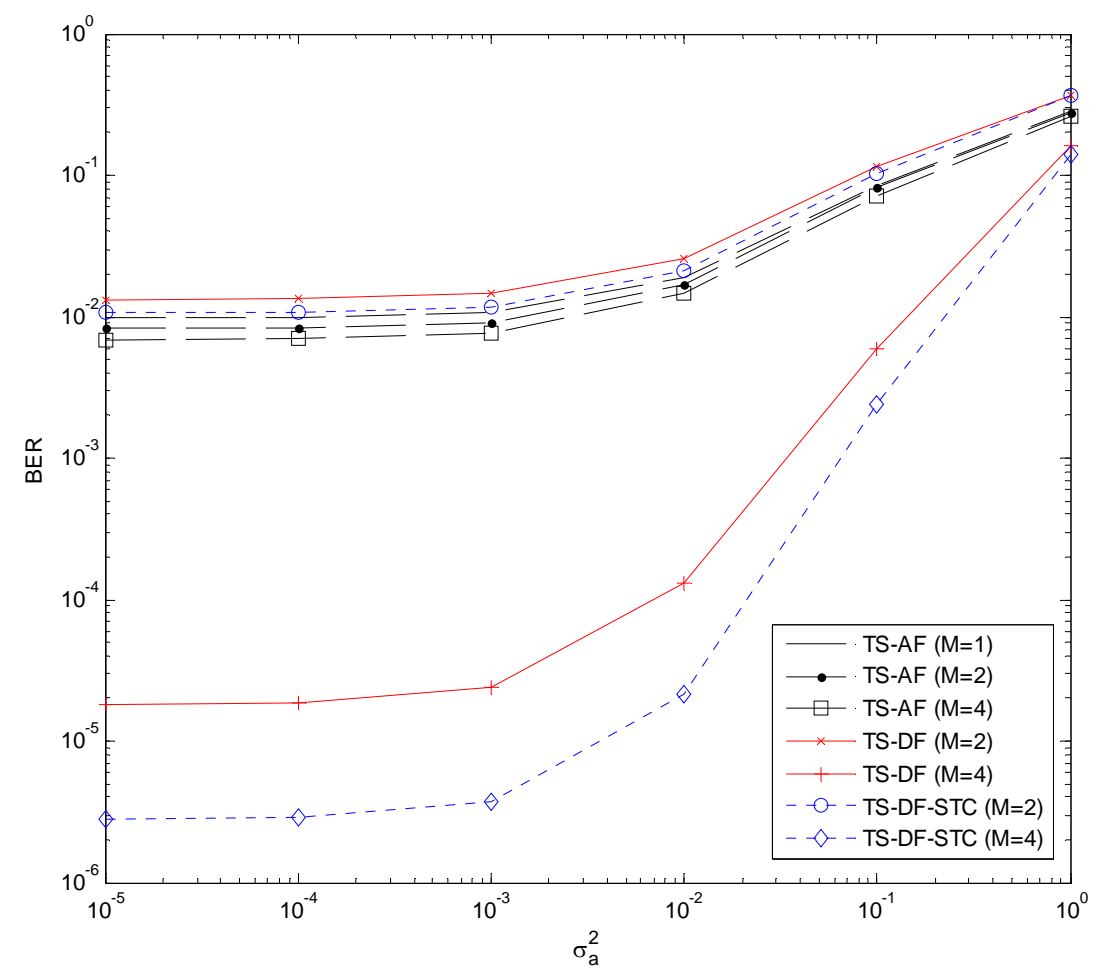

Figure 12. BER versus antenna noise variance $\sigma_{\mathrm{a}}^{2}$ for the TS-DF, TS-DF-STC, and TS-AF schemes (conversion noise variance $\sigma_{\mathrm{c}}^{2}=0.01$ and optimal energy harvesting time).

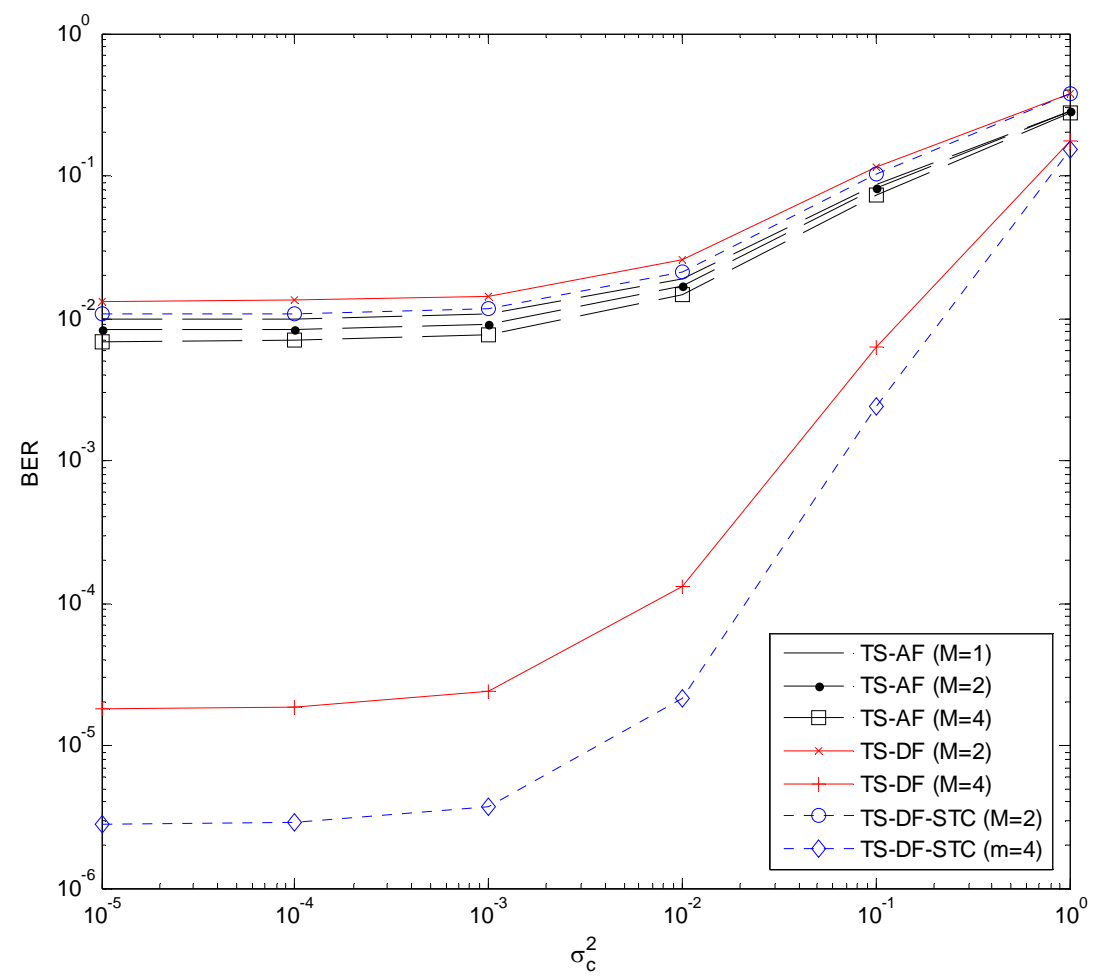

Figure 13. BER versus antenna noise variance $\sigma_{\mathrm{c}}^{2}$ for the TS-DF, TS-DF-STC, and TS-AF schemes (antenna noise variance $\sigma_{\mathrm{a}}^{2}=0.01$ and optimal energy harvesting time). 


\section{Conclusions}

Based on two SWIPT protocols, namely TSR and PSR, we have presented various two-way multi-antenna relaying schemes, where an energy-constrained relay node harvests energy from two source nodes and uses that harvested energy to forward their information. We have compared the BER performance of these SWIPT-based relaying schemes, and have studied the effect of several network parameters, including the number of relay antennas, power splitting ratio, and energy harvesting time. The results have revealed that the benefit of multiple-antenna deployment at the relay depends primarily on the number of antennas (e.g., two antennas versus four antennas), the signal-processing technique (e.g., $\mathrm{AF}$ versus $\mathrm{DF}$ ), and the power allocation strategy (e.g., equal power allocation versus optimal power allocation). In addition, despite the fact that the optimal power allocation is preferable to the equal power allocation, the latter seems an attractive choice for the PS-AF and TS-AF schemes. For the other relaying schemes (i.e., PS-DF, PS-DF-STC, TS-DF, and TS-DF-STC), algorithmic determination of the optimal power splitting ratio and optimal energy harvesting time (see, e.g., [32,33]) could be an interesting direction for future work.

Acknowledgments: This work was supported by Graduate School, Rangsit University.

Author Contributions: Thanaphat Srivantana and Kiattisak Maichalernnukul conceived and designed the experiments; Thanaphat Srivantana performed the experiments; Thanaphat Srivantana and Kiattisak Maichalernnukul analyzed the data; Kiattisak Maichalernnukul contributed reagents/materials/analysis tools; Thanaphat Srivantana and Kiattisak Maichalernnukul wrote the paper.

Conflicts of Interest: The authors declare no conflict of interest.

\section{References}

1. Varshney, L. Transporting information and energy simultaneously. In Proceedings of the IEEE International Symposium on Information Theory, Toronto, ON, Canada, 6-11 July 2008; pp. 1612-1616.

2. Grover, P.; Sahai, A. Shannon meets Tesla: Wireless information and power transfer. In Proceedings of the IEEE International Symposium on Information Theory, Austin, TX, USA, 13-18 June 2010; pp. 2363-2367.

3. Zhou, X.; Zhang, R.; Ho, C.K. Wireless information and power transfer: Architecture design and rate-energy tradeoff. IEEE Trans. Commun. 2013, 61, 4754-4767. [CrossRef]

4. Zhang, R.; Ho, C.K. MIMO broadcasting for simultaneous wireless information and power transfer. IEEE Trans. Wirel. Commun. 2013, 12, 1989-2001. [CrossRef]

5. Huang, K.; Larsson, E.G. Simultaneous information and power for broadband wireless systems. IEEE Trans. Signal Process. 2013, 61, 5972-5986. [CrossRef]

6. Huang, K.; Lau, V.K.N. Enabling wireless power transfer in cellular networks: Architecture, modeling and deployment. IEEE Trans. Wirel. Commun. 2014, 13, 902-912. [CrossRef]

7. Park, J.; Clerckx, B. Joint wireless information and energy transfer in a $k$-user MIMO interference channel. IEEE Trans. Wirel. Commun. 2014, 13, 5781-5796. [CrossRef]

8. Shen, C.; Li, W.; Chang, T. Wireless information and energy transfer in multi-antenna interference channel. IEEE Trans. Signal Process. 2014, 62, 6249-6264. [CrossRef]

9. Nasir, A.A.; Zhou, X.; Durrani, S.; Kennedy, R.A. Relaying protocols for wireless energy harvesting and information processing. IEEE Trans. Wirel. Commun. 2013, 12, 3622-3636. [CrossRef]

10. Yang, D. Wireless information and power transfer: Optimal power control in one-way and two-way relay system. Wirel. Pers. Commun. 2015, 84, 1-14. [CrossRef]

11. Eslamifar, M.; Chin, W.H.; Yuen, C.; Guan, Y.L. Performance analysis of two-step bi-directional relaying with multiple antennas. IEEE Trans. Wirel. Commun. 2012, 11, 4237-4242. [CrossRef]

12. Sanguinetti, L.; D'Amico, A.A.; Rong, Y. A tutorial on the optimization of amplify-and-forward MIMO relay systems. IEEE J. Sel. Areas Commun. 2012, 30, 1331-1346. [CrossRef]

13. Yuan, X.; Yang, T.; Collings, I.B. Multiple-input multiple-output two-way relaying: A space-division approach. IEEE Trans. Inf. Theory 2013, 59, 6421-6440. [CrossRef]

14. Rashid, U.; Tuan, H.D.; Kha, H.H.; Nguyen, H.H. Joint optimization of source precoding and relay beamforming in wireless MIMO relay networks. IEEE Trans. Commun. 2014, 62, 488-499. [CrossRef] 
15. Sheikh, A.; Olfat, A. Diversity achieving schemes for two-way multi-antenna relay networks in Nakagami- $m$ fading. IET Commun. 2015, 9, 1561-1574. [CrossRef]

16. Li, Q.; Zhang, Q.; Qin, J. Beamforming in non-regenerative two-way multi-antenna relay networks for simultaneous wireless information and power transfer. IEEE Trans. Wirel. Commun. 2014, 13, 5509-5520. [CrossRef]

17. Farazi, S.; Brown, D.R., III; Klein, A.G. Power allocation for three-phase two-way relay networks with simultaneous wireless information and power transfer. In Proceedings of the Asilomar Conference on Signals, Systems, and Computers, Pacific Grove, CA, USA, 8-11 November 2015; pp. 812-816.

18. Zhang, Q.; Li, Q.; Qin, J. Beamforming design for OSTBC-based AF-MIMO two-way relay networks with simultaneous wireless information and power transfer. IEEE Trans. Veh. Technol. 2016, 65, 7285-7296. [CrossRef]

19. Dohler, M.; Li, Y. Cooperative Communications: Hardware, Channel \& PHY; Wiley: Chichester, UK, 2010; pp. 20-25.

20. Fragouli, C.; Le Boudec, J.Y.; Widmer, J. Network coding: An instant primer. ACM SIGCOMM Comput. Commun. Rev. 2006, 36, 63-68. [CrossRef]

21. Tarokh, V.; Jafarkhani, H.; Calderbank, A.R. Space-time block codes from orthogonal designs. IEEE Trans. Inf. Theory 1999, 45, 1456-1467. [CrossRef]

22. Simon, M.K.; Alouini, M.-S. Digital Communication over Fading Channels: A Unified Approach to Performance Analysis; Wiley: New York, USA, 2000.

23. Gradshteyn, S.; Ryzhik, I.M. Table of Integrals, Series, and Products; Academic Press: New York, USA, 2000.

24. Arti, M.K.; Mallik, R.K.; Schober, R. Channel estimation and decoding of OSTBC in two-way AF MIMO relay networks. In Proceedings of the IEEE Vehicular Technology Conference, Las Vegas, NV, USA, 2-5 September 2013; pp. 1-5.

25. Zhao, Q.; Zhou, Z.; Li, J.; Vucetic, B. Joint semi-blind channel estimation and synchronization in two-way relay networks. IEEE Trans. Veh. Technol. 2014, 63, 3276-3293. [CrossRef]

26. Jiang, Y.; Varanasi, M.K.; Li, J. Performance analysis of ZF and MMSE equalizers for MIMO systems: An in-depth study of the high SNR regime. IEEE Trans. Inf. Theory 2011, 57, 2008-2026. [CrossRef]

27. Proakis, J.G.; Salehi, M. Digital Communications, 5th ed.; McGraw-Hill: New York, USA, 2008.

28. Papoulis, A. Probability, Random Variables and Stochastic Processes, 3rd ed.; McGraw-Hill: New York, USA, 1991.

29. Siriwongpairat, W.P.; Olfat, M.; Liu, K.J.R. Performance analysis and comparison of time-hopping and direct-sequence UWB-MIMO systems. EURASIP J. Appl. Signal Process. 2005, 2005, 328-345. [CrossRef]

30. Maichalernnukul, K.; Zheng, F.; Kaiser, T. Performance analysis of a MIMO-RFID system in Nakagami- $m$ fading channels. Ann. Telecommun. 2016, 71, 151-156. [CrossRef]

31. Rayleigh_Distribution. Available online: https://en.wikipedia.org/wiki/Rayleigh_distribution (accessed on 5 January 2017).

32. Shi, Q.; Liu, L.; Xu, W.; Zhang, R. Joint transmit beamforming and receive power splitting for MISO SWIPT systems. IEEE Trans. Wirel. Commun. 2014, 13, 3269-3280. [CrossRef]

33. Vu, Q.-D.; Tran, L.-N.; Farrell, R.; Hong, E.-K. An efficiency maximization design for SWIPT. IEEE Signal Process. Lett. 2015, 22, 2189-2193. [CrossRef]

(C) 2017 by the authors. Licensee MDPI, Basel, Switzerland. This article is an open access article distributed under the terms and conditions of the Creative Commons Attribution (CC BY) license (http://creativecommons.org/licenses/by/4.0/). 